\title{
Article
}

\section{Modeling and Simulation of Sky Survey}

\author{
Kun $\mathrm{Hu}^{1, t, \mp(\mathbb{D}, \text { Hongfei Wang }}{ }^{2, *}$, Wenzhao Zhang ${ }^{3, \ddagger}$, Yuohua $\mathrm{Xu}^{4, \ddagger}$ and Shengmao $\mathrm{He}^{2, \ddagger}$ \\ 1 University of Chinese Academy of Sciences, Beijing 100049, China; hukun19@mails.ucas.ac.cn \\ 2 Key Laboratory of Space Utilization, Technology and Engineering Center for Space Utilization, \\ Chinese Academy of Sciences, Beijing 100094, China; heshengmao13@csu.ac.cn \\ 3 Astronomy Department, Beijing Normal University, Beijing 100875, China; wenzhaozhang@bnu.edu.cn \\ 4 CAS Key Laboratory of Space Astronomy and Technology, National Astronomical Observatories, \\ Beijing 100101, China; yhxu@nao.cas.cn \\ * Correspondence: whf@csu.ac.cn \\ + Current address: No. 9, Dengzhuang South Road, Haidian District, Beijing 100094, China. \\ $\ddagger$ These authors contributed equally to this work.
}

check for

updates

Citation: Hu, K.; Wang, H.; Zhang, W.; Xu, Y.; He, S. Modeling and Simulation of Sky Survey. Appl. Sci. 2021, 11, 7584. https://doi.org/ 10.3390/app11167584

Academic Editor: Aki Mikkola

Received: 19 July 2021

Accepted: 16 August 2021

Published: 18 August 2021

Publisher's Note: MDPI stays neutral with regard to jurisdictional claims in published maps and institutional affiliations.

Copyright: (C) 2021 by the authors. Licensee MDPI, Basel, Switzerland. This article is an open access article distributed under the terms and conditions of the Creative Commons Attribution (CC BY) license (https:/ / creativecommons.org/licenses/by/ $4.0 /)$.

\begin{abstract}
Sky surveys with wide and deep field are the key direction of international astronomy research for the next decades. It is worthwhile to study how to design a sky survey algorithm to obtain the maximum scientific output in a limited period of observation. In this paper, the modeling and simulation of a wide and deep sky survey mission are presented using a Staged Design Algorithm (SDA), which takes into account the inefficient time periods and difficult-to-observe sky areas. In order to ensure the effective completion of the long-time survey observation tasks in large sky areas, a two-stage scheduling algorithm is designed. Firstly, the inefficient time periods and difficult observation areas are scheduled, and then the overall observation is carried out. The prearranged schedule is arranged when inefficient time periods or difficult areas are encountered during the overall arrangement. The simulation results are verified on the basic data of the China Space Station Telescope (CSST), and the obtained simulation result is three years ahead of the target of the telescope design to complete the wide and deep sky survey of 15,000 deg ${ }^{2}$. The design ideas in this paper not only have good results for sky survey observation but also can be extended to similar satellite Earth observation mission planning.
\end{abstract}

Keywords: sky surveys; system simulation; mission planning; staged design algorithm

\section{Introduction}

Sky surveying has given a huge boost to the development of astronomy [1,2], and there are many internationally renowned surveys, such as Supernovae observations [3-6], Sloan Digital Sky Survey [7-9], Legacy Survey of Space and Time (LSST) [10-12], Hubble Space Telescope (HST) [13-15], Euclid [16-18], Wilkinson Microwave Anisotropy Probe (WMAP) [19-21], and Planck [22,23], which have made a number of major scientific discoveries in astronomy, such as the discovery of the accelerating expansion of the universe [3]. Table 1 shows a comparison of the specification parameters of CSST, LSST, Euclid, and WFIRST [24-26] with information from Zhan [27].

In 2024, China will launch the China Space Station Telescope (CSST) [27,28]. It will be launched at the Wenchang launch site using the ChangZheng 5B launch vehicle. A wide and deep optical survey will be carried out to complete a 15,000 deg ${ }^{2}$ sky area, including deep multicolor imaging observations, very deep multicolor imaging observations, slitless spectroscopic observations, and deep slitless spectrography observations [8,27].

The mission planning is a very complex problem, and there are many factors affecting the mission strategy, including the orbital motion of satellites; the influence of the Sun, Moon, and Earth light on the observations; the need to meet the energy supply requirements of the vehicle's solar panel in the sunlit region; and the influence of the Earth South Atlantic Anomaly (SAA) region on the equipment during the ground orbit operation. The complex 
constraints and wide sky area factors bring great challenges to the existing optimization algorithms to solve the sky survey observation models.

Table 1. Specifications of LSST, WFIRST, Euclid, and the suggested wide and deep survey on board the CSST [27].

\begin{tabular}{|c|c|c|c|c|c|}
\hline & CSST & LSST & \multicolumn{2}{|c|}{ Euclid } & WFIRST \\
\hline Caliber & $\geq 2$ & 8.4 & \multicolumn{2}{|c|}{1.2} & 1.3 \\
\hline Wavelength Range & $200-1000$ & $320-1050$ & 550-920 & $1000-2000$ & $760-2000$ \\
\hline Effective Field of View & $\geq 1$ & 10 & 0.56 & 0.55 & 0.85 \\
\hline Image Quality & $\leq 0.15$ & 0.7 & 0.2 & 0.6 & 0.3 \\
\hline Relative Skywatching Speed & $\geq 3.1$ & 7.3 & 1 & $\mathrm{NaN}$ & 0.7 \\
\hline Sky Survey Area & $1 \overline{5}, 000$ & 20,000 & 15,000 & $\mathrm{NaN}$ & 14,000 \\
\hline Band Number & 6 & 6 & 1 & 3 & 4 \\
\hline Availability of Spectrum & Yes & No & No & Yes & Yes \\
\hline
\end{tabular}

The survey observation of large space astronomy telescopes is a complex task [29], and advanced planning and operation control technology is the basis for the efficient completion of the task. The space astronomy observation telescope will be constrained by various factors in the process of a sky survey observation. In order to improve the observation efficiency, overall mission planning and optimal allocation of resources are required for the observation sequence of different target sky areas. The entire optimization of the life-cycle observation plan while in orbit operation will be performed to enhance efficiency and maximize the value of scientific applications. Figure 1 shows the planning diagram of the sky survey observation mission.

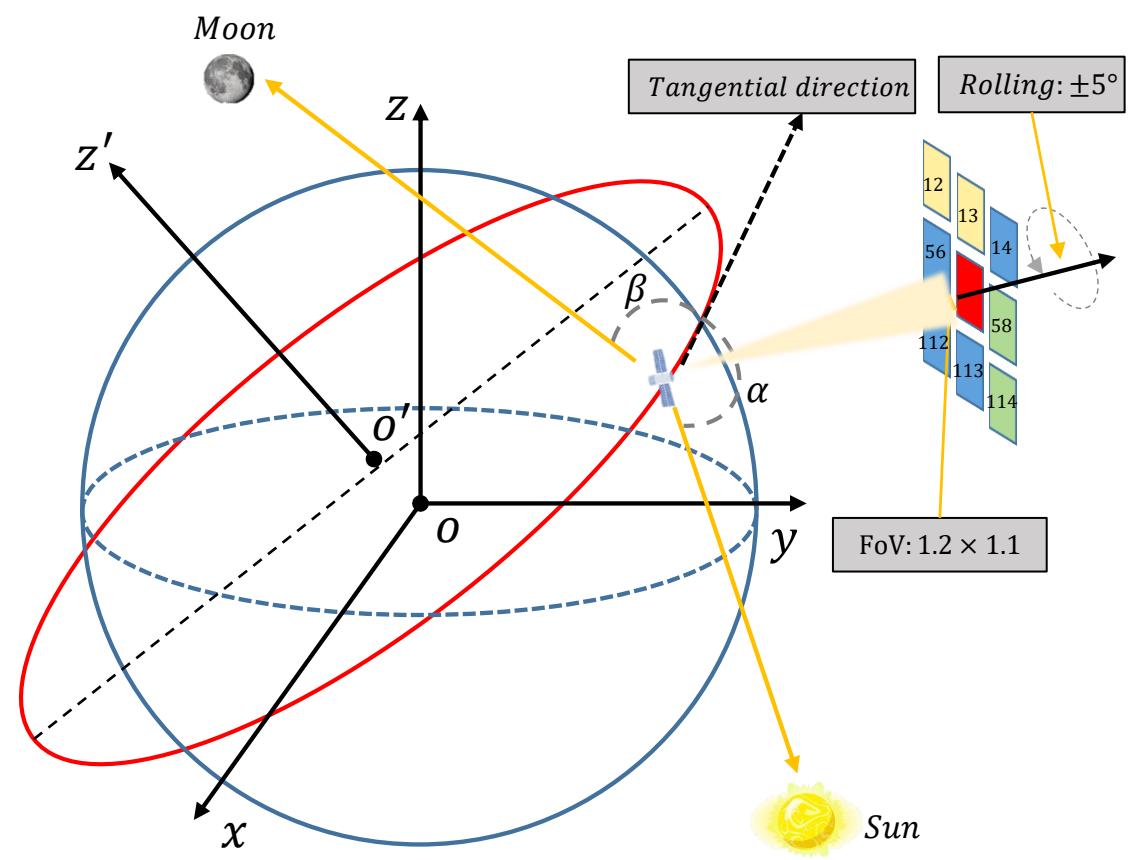

Figure 1. Diagram of the sky survey mission planning. The survey telescope needs to satisfy certain observing constraints during on-orbit observations, such as the angle constraints between the sunlight $(\alpha)$, lunar light $(\beta)$, and the observation pointing, etc., shown in the figure. Different colors in the sky zone represent different levels of importance (degree of value) of the observed sky zone. For example, there are extremely deep sky zones, seamless spectral sky zones, etc. The telescope can only be rotated parallel to the orbital plane normal ( $Z^{\prime}$ axis) during the search for the observing sky area. The red square is the area currently under observation. The telescope can only observe one sky area at a time and needs to keep observing for a fixed period of time to complete a single observation. 


\section{Related Work}

Research in sky survey telescopes has focused on models, algorithms, and planning systems. In this section, the research is presented from those aspects. Since the research related to models and algorithms is inseparable, the models and algorithms are presented together.

\subsection{Models and Algorithms}

Most of the sky survey planning models are multi-objective optimization models $[30,31]$ and constraint satisfaction models [32]. Heuristic algorithms are widely used in the optimization model solving for sky survey observations, such as genetic algorithms [30] and greedy algorithms [33].

Liu et al. [30] focused on the planning of the sky survey observation mode and established an intelligent planning model for the trajectory of the celestial sphere star image points. Their main objectives are: (i) to add as little attitude maneuver control as possible; (ii) to use as little energy as possible; and (iii) to achieve as many sky area scans as possible. Using a combination of orbital surface approach and orbital motion, the scanning of the celestial sphere is achieved through intelligent control of the scanning mission. While the attitude maneuver control demand on the satellite is only two times (roll angle $\pm 30^{\circ}$ transitions), these two maneuvers divide the whole sky survey plan into three major time periods, each with an orbital approach of $360^{\circ}$ within the duration of the time period, allowing the scanning of the entire sky area. The main constraint addressed by the planning algorithm is the energy deficit problem. To solve this problem, a multi-objective genetic algorithm is used.

Jin et al. [31] have studied, in detail, modeling the dynamics of spacecraft and cruise payloads, the constraints on sky observations, the sky area coverage model, and the payload mission planning model, respectively. The main scientific goal of conducting a 30,000 deg 2 survey in four years can be achieved.

Wang et al. [32] converted the survey observation scheduling problem into a constrained optimization problem and linearized the objective and constraint functions by using $0-1$ decision variables and solved them using a well-established algorithm. However, the example part of the survey test data only has 30 consecutive time windows and relatively few candidate sky areas. The large-scale and long-time survey problem has not been studied.

In addition to research on space-based survey planning, there is also research on survey planning algorithms based on ground-based telescopes. Although they differ in terms of observation environment and observation mode, there are mutual references in optimization algorithms. Huang et al. [33] established a mathematical model for the Hard X-ray Modulation Telescope (HXMT) long-term mission planning problem by analyzing the optimization objectives and constraints of the problem according to the characteristics of HXMT satellites. The objective of the model is to make the observation efficiency and mission rank the highest. Based on this, a greedy algorithm mixed with a genetic algorithm is proposed as a composite solution method, and the effectiveness of the method is verified through simulation. The algorithm was tested using observation missions generated from Suzaku satellite data, and the results showed that the algorithm was effective in solving the long-term mission planning problem for the HXMT satellite, making full use of satellite resources and maximizing scientific output while meeting the constraints.

\subsection{Sky Survey Planning Systems}

The sky survey planning system can bring together complete task setup, optimization scheduling, result output, and visual presentation in one software. It is extremely convenient for scientists who are not in the direction of algorithmic research to schedule their sky survey missions. Therefore, various spacefaring nations have developed related software and applied it in actual spacecraft launch missions. Table 2 summarizes some of the more representative software systems, according to Wang et al. [32] and Amiaux et al. [17]. 
Table 2. Several widely used planning systems.

\begin{tabular}{ccc}
\hline Planning System Name & Main Algorithm & Notes \\
\hline Palomar Transient Factory [34] & $\begin{array}{c}\text { Automated queue scheduler/ } \\
\text { Shortsighted strategy }\end{array}$ & $\begin{array}{c}\text { Ability to perform real-time } \\
\text { scheduling optimization }\end{array}$ \\
\hline Remote Telescope System 2nd Edition [35] & Value function/genetic algorithm & Code Open Source \\
\hline Spike planning and scheduling software [36] & Neural networks & Hubble/AXAF/SIRTF \\
\hline Sky Survey Planning Tool [17] & No explicit information & Herchel/Euclid \\
\hline Multi-User Scheduling Environment [29] & No information available & JWST/Cassini/Cluster WBD
\end{tabular}

Spike planning and scheduling software [36], Sky Survey Planning Tool [17], and Multi-User Scheduling Environment [29] are applied in real satellite planning tasks with excellent optimization results. The Euclid telescope was able to achieve the science goal of covering an area of 15,500 deg in 5.5 years, which also includes a $40 \mathrm{deg}^{2}$ deep-field observation area. With the Euclid Sky Survey Planning Tool (ESSPT), which was used by the Herschel satellite, Euclid is able to prioritize the observation of the areas of highest scientific value in the first year [17].

In summary, there is still relatively little research on large-scale survey observations at home and abroad. Although the more mature Hubble telescope has both short-term and long-term planning algorithms, there are few detailed papers describing the algorithms in detail, and most of the research is carried out on simple survey missions, taking into account relatively few practical factors that do not have practical engineering use value. Thus, research on wide and deep survey algorithms becomes very urgent. To address the problem of the above research not being detailed and the model being in a black box state, this paper gives a wide and deep sky survey algorithm based on stage processing. Our main contributions are summarized as follows:

- Various constraint analyses of the sky survey planning design are given in detail, which are divided into observation constraints and orbit constraints, and the multiobjective optimization model of the sky survey problem is established.

- The Staged Design Algorithm (SDA) is designed to prioritize the time efficiency and the difficulty of observation sky areas to maximize the efficiency of long-time observations.

- The model is solved using real data, and the obtained results are three years ahead of the predicted completion date of the sky survey mission, indicating that the algorithm performs well.

The article is organized as follows. Section 3 expresses the specific problem of sky survey planning and analyzes the corresponding constraints and objective functions. Section 4 shows the Staged Design Algorithm (SDA) for solving the sky survey optimization model. Section 5 gives the results of the sky survey observations with real data and gives the comparison results of the algorithm for the efficiency of the sky survey observations with different parameter settings. In Section 6, a detailed summary of the paper is exhibited. In the last Section 7, the conclusions of this paper and directions for future research are given.

\section{Proposed Methods}

In order to gain a clearer understanding of the sky survey problem, this section provides a detailed analysis of the sky survey mission, introduces some assumptions and simplifications, analyzes the constraints of the model, establishes the objective function, and introduces the Staged Design Algorithm.

We develop a multi-objective and multi-constraint planning model to complete a wide and deep sky survey observation mission and to satisfy the basic and main requirements of the observation. First, we build a multi-objective mission planning model with two objective functions and three constraints. Pre-scheduling is achieved by counting inefficient time periods and difficult observation sky areas. Then, a greedy algorithm is used to determine the observation sky area with the maximum weight, which leads to the sky 
survey observation scheme for the sky area. The flow chart of the sky survey planning proposed in this paper is shown in Figure 2.

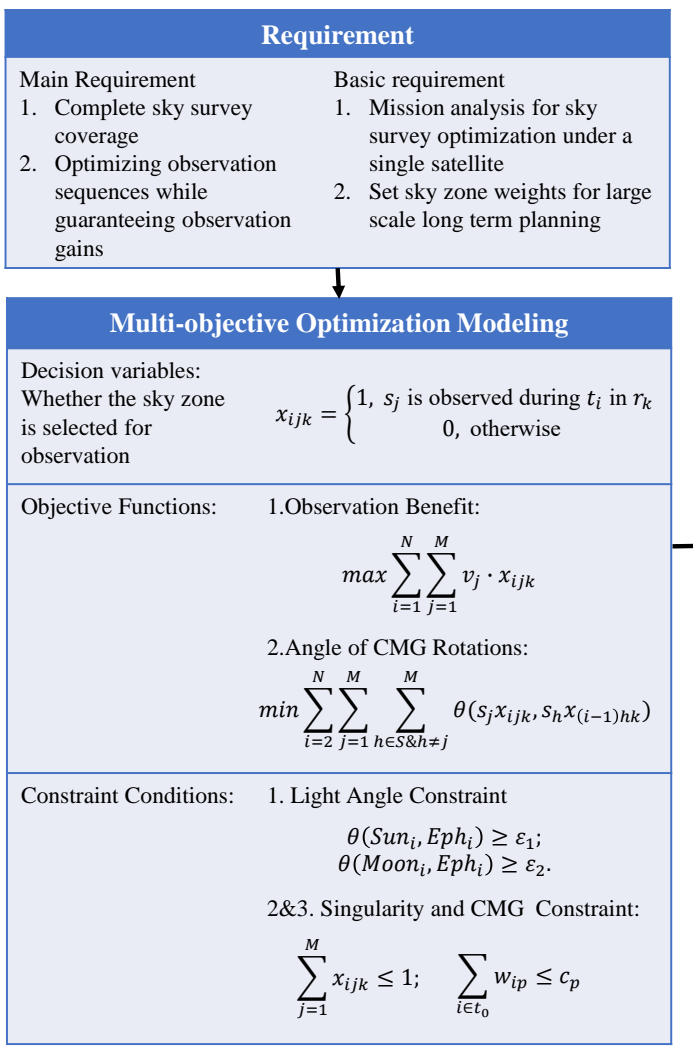

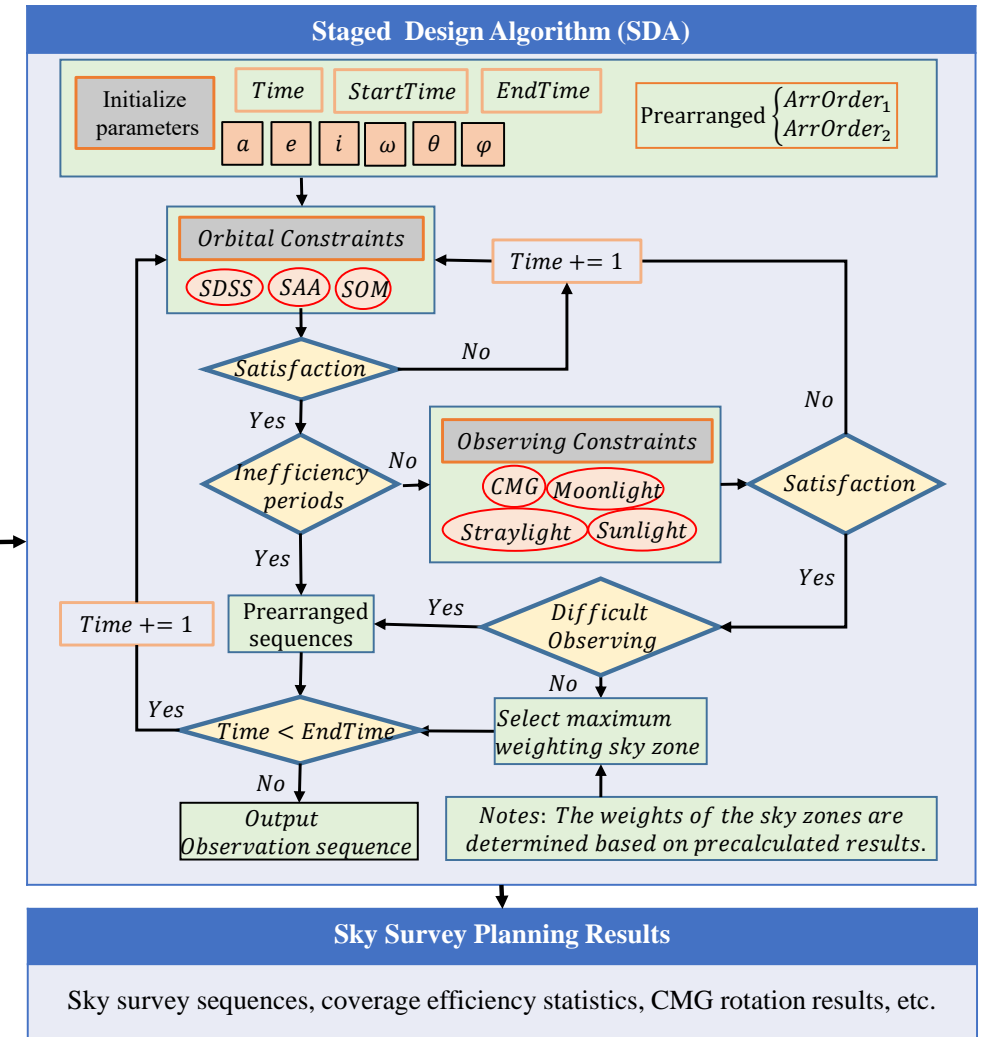

Figure 2. The flow chart of the wide and deep sky survey observation mission in this paper.

\subsection{Assumptions and Simplifications}

The sky survey scheduling optimization is characterized by many variables and many constraints, and the optimization model contains a huge solution space, which is a nondeterministic polynomial hard problem [37]. In order to simplify the model by mainly studying the important constraints and ignoring the secondary constraints, this paper makes assumptions about some of the constraints in the problem to facilitate our study of the problem.

- Only the smallest sky area is accurately observed for each observation, and there is no misalignment of observations, which means that the satellite CMG can be accurately positioned on the smallest sky area block of the observation;

- It assumes satellites meet data storage and total energy limits (CMG energy limits are not included);

- Assuming that the steady image time is already within the CMG rotation time, no additional steady image time is calculated;

- It is assumed that there are no sudden events that affect camera imaging during the simulation time, such as high-energy rays from solar activity.

\subsection{Advance Definition}

Before building the model and analyzing the constraints, we define and explain some of the symbols used to facilitate the model building later. In this paper, we refer to two more established papers on the definition of model notation [32,38]. In order to be more concise and uniform in describing the model and algorithm process below, the symbols used and the specific descriptions are listed in Table 3. 
Table 3. Symbols used and specific instructions.

\begin{tabular}{cc}
\hline Symbols & Detailed Description \\
\hline$N, M$ & Total number of time windows and sky areas \\
$\alpha$ & Tangential angle between sunlight and satellite \\
$\beta$ & Tangential angle between lunar light and satellite \\
$T=\left\{t_{i}, i=1,2, \ldots, N\right\}$ & Set of observed time windows \\
$S=\left\{s_{j}, j=1,2, \ldots, M\right\}$ & Set of Sky area division \\
$R=\left\{r_{k}, k=1,2, \ldots, K\right\}$ & Set of observed orbital period \\
$C=\left\{c_{p}, p=1,2, \ldots, 8\right\}$ & Set of CMG rotation times \\
$v_{j}$ & Weighting factor of the $j$-th sky area \\
$x_{i j k}=\{0,1\}$ & $s_{j}$ is observed during $t_{i}$ in $r_{k}$ \\
$r$ & The unit radius \\
$\omega_{i p}$ & The $p$-th CMG rotation angle at the $i$-th time window \\
Low & Inefficient observation time period \\
$D i f S k y$ & Difficult to observe sky areas \\
$A=\{E p h, S u n$, Moon $\}$ & Satellite, Sun, and Moon ephemeris data \\
$\gamma_{i}(i=1,2)$ & Threshold parameters of efficiency and difficulty \\
$\epsilon_{i}(i=1,2)$ & Threshold parameters of sunlight and moonlight \\
$S t, E t$ & Start time and end time of sky survey \\
Oc $c=\{S A A, S O M, S D S S\}$ & Set of orbital constraints \\
$T c=\left\{C_{1}, C_{2}, C_{3}\right\}$ & Set of observation constraints \\
$\delta$ & Zenith angle \\
\hline
\end{tabular}

Since the sky survey observation is performed by point gazing, which requires a long time to observe a small block of sky area, there is a consumption of camera rotation time in the actual operation, which is called the altitude camera time constraint in this paper. The altitude maneuver time depends on the turning angle. Different corners consume different amounts of time, with the following constraints.

$$
\text { TimeCMG }=\left\{\begin{array}{cc}
45, & \delta \leq 1^{\circ} \\
100, & 1^{\circ}<\delta \leq 20^{\circ} \\
170, & 20^{\circ}<\delta \leq 45^{\circ} \\
170+(\delta-50) / 0.35, & 45^{\circ}<\delta .
\end{array}\right.
$$

The time indicated above includes the readout time of the CCD and the stabilization time of the camera and is greater than $45^{\circ}$ when using a speed of $0.35(\%)$ for time computation.

\subsection{Analysis of Orbital Constraints}

Orbital constraints refer to the exclusion of time periods when observations can not be made prior to the official sky survey, including the South Atlantic Anomaly region, space station docking, etc. After these times are excluded, the remaining time is the optimized observation time period, and what needs to be optimized is which region of the sky to observe at each moment.

South Atlantic Anomaly (SAA): As is well known, SAA is a zone that receives and transmits significant amounts of radiation from outer space [39]. Space telescopes may experience instrument malfunctions and communication errors, as well as a large amount of particles forming background noise that reduces the signal-to-noise ratio of the images. Therefore, when a survey passes through this region, the survey module should stop observing (standby or shutdown) unless there is a special observing need.

Satellite Orbit Maintenance (SOM): The station performs orbital maintenance every 80 days for 12 orbital periods and is not observable during orbital maintenance.

Satellite Docking with Space Station (SDSS): The telescope is currently designed to fly with the Chinese space station, so it needs to dock with the Chinese space station periodically to complete status checking and some necessary maintenance, etc. Each space station docking takes between 20 days and 3 months. It is completely invisible throughout this time. 


\subsection{Decision Variables}

The decision variable of the model in this paper is set to the observation status of the telescope at any moment. Let the matrix $X=\left(x_{i j k}\right)_{N \times M \times K}$ denote the decision matrix of the assignment problem, where element $x_{i j k}$ equals " 1 " if the $j$-th sky zone is observed during time window $t_{i}$ in the $k$-th time period; otherwise, $x_{i j k}$ is 0 , given in Equation (2). There are various reasons for not being in observation, and the decision variable is 0 as long as any of the constraints are not satisfied, e.g., SAA, docking with the space station, or orbit maintenance, etc.

$$
x_{i j k}=\left\{\begin{array}{c}
1, \text { if the } s_{j} \text { is observed during time window } t_{i} \text { in the } r_{k} \\
0, \text { otherwise, }
\end{array}\right.
$$

where the values of $k$ in this paper range from $1,2, \ldots, K$, and the range of values of $i$ is the lifetime of the satellite. The interval of time $i$ is chosen differently in short and long planning, with longer intervals in long planning tasks.

\subsection{Objective Function}

In this paper, we consider the objective function from two main aspects. The first one is to introduce the fitness function for evaluating the importance and observation value of the observed sky area, which is described in detail in Wang et al. [32]. The problem of this method is that the observation value needs to be set in advance for the sky area, and the setting of the observation area has different opinions from different scientists, making the setting of the observation value often biased. Another way is to target the observation time and the number of altitude adjustments (energy consumption) for the sky survey. This approach minimizes the survey observation time and energy output from the actual situation of the satellite and builds a multi-objective optimization model. The problem is that the altitude adjustment of the satellite does not need to be minimized, but only the energy requirement of the observation needs to be satisfied. There is a detailed description in Liu et al. [30]. Combining the objective functions of the two papers, this paper takes maximizing the observation benefit and minimizing the angle of CMG rotations as the objective functions.

Observation Benefit: Maximizing the observational benefit means that high-value sky areas are given the highest possible priority for observation and setting the observational priority of sky areas.

$$
O_{1}=\operatorname{Max} \sum_{i=1}^{N} \sum_{j=1}^{M} v_{j} \cdot x_{i j k}, \quad \forall t_{i} \in \boldsymbol{T}, \forall r_{k} \in \boldsymbol{R} .
$$

CMG Rotations: Since the CMG rotation process not only needs to consume energy but also needs to consume the observation time, in order to complete the target cruise faster and better, the objective function is set to the angle and minimum of CMG rotations.

$$
\mathrm{O}_{2}=\min \sum_{i=2}^{N} \sum_{j=1}^{M} \sum_{h \in S \& h \neq j}^{M} \theta\left(s_{j} x_{i j k}, s_{h} x_{(i-1) h k}\right), \quad \forall t_{i} \in \boldsymbol{T}, \forall r_{k} \in \boldsymbol{R} .
$$

where $\theta(S, O)$ is the formula for calculating the rotation angle between the position coordinates of the satellite at any moment $S\left(\right.$ Lat $\left._{1}, \operatorname{Lon}_{1}\right)$ and the position coordinates of the observation point $O\left(\mathrm{Lat}_{2}, \mathrm{Lon}_{2}\right)$ according to $[40,41]$.

$$
\begin{gathered}
f(S, O)=\arcsin \left(\sqrt{\sin \left(\frac{\text { Lat }_{2}-\text { Lat }_{1}}{2}\right)^{2}+\cos \left(\text { Lat }_{1}\right) \cdot \cos \left(\text { Lat }_{2}\right) \cdot \sin \left(\frac{\text { Lon }_{2}-\text { Lon }_{1}}{2}\right)^{2}}\right), \\
\theta(S, O)=2 \cdot r \cdot f(S, O) \times 180^{\circ} / \pi
\end{gathered}
$$


where $f(S, O)$ stands for the function of calculating the angle between $S$ and $O ; r$ in the formula represents the unit radius, as $r=1$; and the unit of position coordinates $\left(\right.$ Lat $_{1}$, Lon $\left._{1}\right)$ and $\left(\right.$ Lat $_{2}$, Lon $\left._{2}\right)$ is the unit radius.

\subsection{Constraints}

In order to more accurately reflect the real conditions of a survey telescope in space while taking into account the complexity of the model, this section describes the use of several important constraints on the model in this paper.

Light Angle: The stray light constraints considered in this paper are divided into two kinds, sunlight and moonlight. Stray light from the Sun and Moon into the hood will increase the noise and seriously affect the quality of imaging. It is necessary to make the angle between the sunlight, moonlight, and the field of view meet a certain angle for imaging.

$$
C_{1}: \alpha=\theta\left(\operatorname{Sun}_{i}, E p h_{i}\right) \geq \omega_{1}, \beta=\theta\left(\text { Moon }_{i}, E p h_{i}\right) \geq \omega_{2}, \quad \forall t_{i} \in \mathbf{T} .
$$

Singularity Constraint: It is necessary to constrain any time window to observe no more than one sky zone. Thus, we have the constraint $C_{1}$ :

$$
C_{2}: \sum_{j=1}^{M} x_{i j k} \leq 1, \quad \forall t_{i} \in \boldsymbol{T}, \forall r_{k} \in \boldsymbol{R}
$$

CMG Constraint: As the CMG rotation process needs to consume capacity, the larger the rotation angle, the more energy consumed. The number of maneuvers of different angles of the monorail rotation needs to be limited to ensure the normal operation of the CMG. Let $C=\left\{c_{p}, p=1,2, \ldots, 8\right\}$ be a CMG rotation times set.

$$
C_{3}: \sum_{i \in t_{0}} \omega_{i p} \leq \mathrm{c}_{p}, \quad p=1,2, \ldots, 8,
$$

where $t_{0} \in\left[\left\lfloor t_{i} / T\right\rfloor \times T, t_{i}\right],\lfloor *\rfloor$ stands for rounding down to the nearest integer for $* ; t_{i}$ is the current time; and $\omega_{i p}$ represents the $p$-th CMG rotation angle at the $i$-th time window.

\section{Design of the Model Solving Algorithm}

According to the related work section, most of the existing sky survey planning algorithms are based on heuristics, and the most used are genetic algorithms [30,32]. Genetic algorithms still have a large advantage in solving small-scale and short-time survey observation tasks, but they are strained for wide and long-time sky survey observation tasks. In this paper, we use the Staged Design Algorithm based on the idea of greedy algorithm from the constraints of the task, which can handle the observation difficulties in long-time sky survey tasks. The observation times are reserved in advance for the difficult observation sky areas, so as to solve the problem of consuming a lot of resources to observe a few sky areas individually at the late stage of observation. The Staged Design Algorithm is divided into three steps: statistical phase (observation efficiency statistics and observation difficulty statistics), prearrangement stage, and overall arrangement stage.

\subsection{Overview of Stage Design Algorithm}

Figure 2 illustrates the flow chart of the Staged Design Algorithm proposed in this paper. Specifically, first, the initialization parameters, the start time, the end time, the number of orbital roots, and the statistics of inefficiency periods (Low) and difficult observing sky areas (DifSky) are prearranged in advance according to Algorithms 1 and 2. The simulation time is initialized, and the orbit constraint is checked. Under the condition that the orbit constraint is satisfied, it is checked whether it belongs to the prearranged low efficiency time period. If it belongs to this, then the prearranged scheme of low efficiency for observation directly follows. Otherwise, it judges whether the observation constraint 
is satisfied. After it does not satisfy, the time of simulation is increased and returned. If it is satisfied, then it check whether it belongs to the difficult observation sky area. If yes, then it executes the prearrangement. If it does not satisfy, it finds the sky zone with the largest weight factor for observation. Finally, the simulation time is judged to be over. If it is met, the observation result is output. Otherwise the time is increased, and the observation continues.

\subsection{Observation Efficiency Statistics}

Through the constraint analysis in Section 3.6, we are able to find that the main factors affecting the observation efficiency are sunlight and lunar light, so these two constraints are mainly considered in the preliminary statistics of the observation efficiency. We know that the threshold value for judging the inefficient time period is set differently, and the final optimized results differ. The threshold should be set in combination with the orbital parameters of the satellite and also the observation needs. A detailed analysis of the effect of different parameters on the optimization results will be given later in the experimental section. The steps to calculate the inefficient time period are shown in Algorithm 1. For inefficient observation time periods, we perform prearranged observation tasks shown in Figure 2.

\subsection{Observation Difficulty Statistics}

Different sky areas at different locations have different observation difficulties. According to the known experience of optimization algorithms, the difficult sky areas may have the possibility of not being observed later, so the sky areas with higher observation difficulties should be counted for advance observation arrangements. Algorithm 2 shows the result of observing the difficulty statistics and prioritizing the sky areas with high difficulty. Similar to $\gamma_{1}$ in Algorithm 1, here, $\gamma_{2}$ is also a parameter set according to the satellite orbit.

\subsection{Prearrangement Algorithm}

After the statistics of inefficient time periods and difficult observation sky areas, it is necessary to prearrange the program to both cases to ensure that the inefficient time periods and difficult observation sky areas can be arranged effectively during the overall sky survey arrangement. Algorithm 3 gives the arrangement algorithm for the inefficient time period, and we obtain the arrangement sequence ArrOrder ${ }_{1}$. Since the arrangement algorithm for the difficult sky area is similar to it, we do not show it in detail, and we obtain the arrangement sequence ArrOrder 2 for the difficult sky area correspondingly.

\subsection{Overall Arrangement Algorithm}

The scheduling process is time-based, and since the time periods that cannot be observed have been eliminated in advance, the time periods for this part of the optimization schedule are those in which all the sky areas can be observed. A greedy algorithm is used to determine the observation sequence with a fixed optimization time interval. Specifically, the greedy algorithm is used to select the sequence of observed sky areas. The one with the highest factor weight is selected for priority observation. Algorithm 4 details the completion of the planning process, and the corresponding process is shown in Figure 2. 

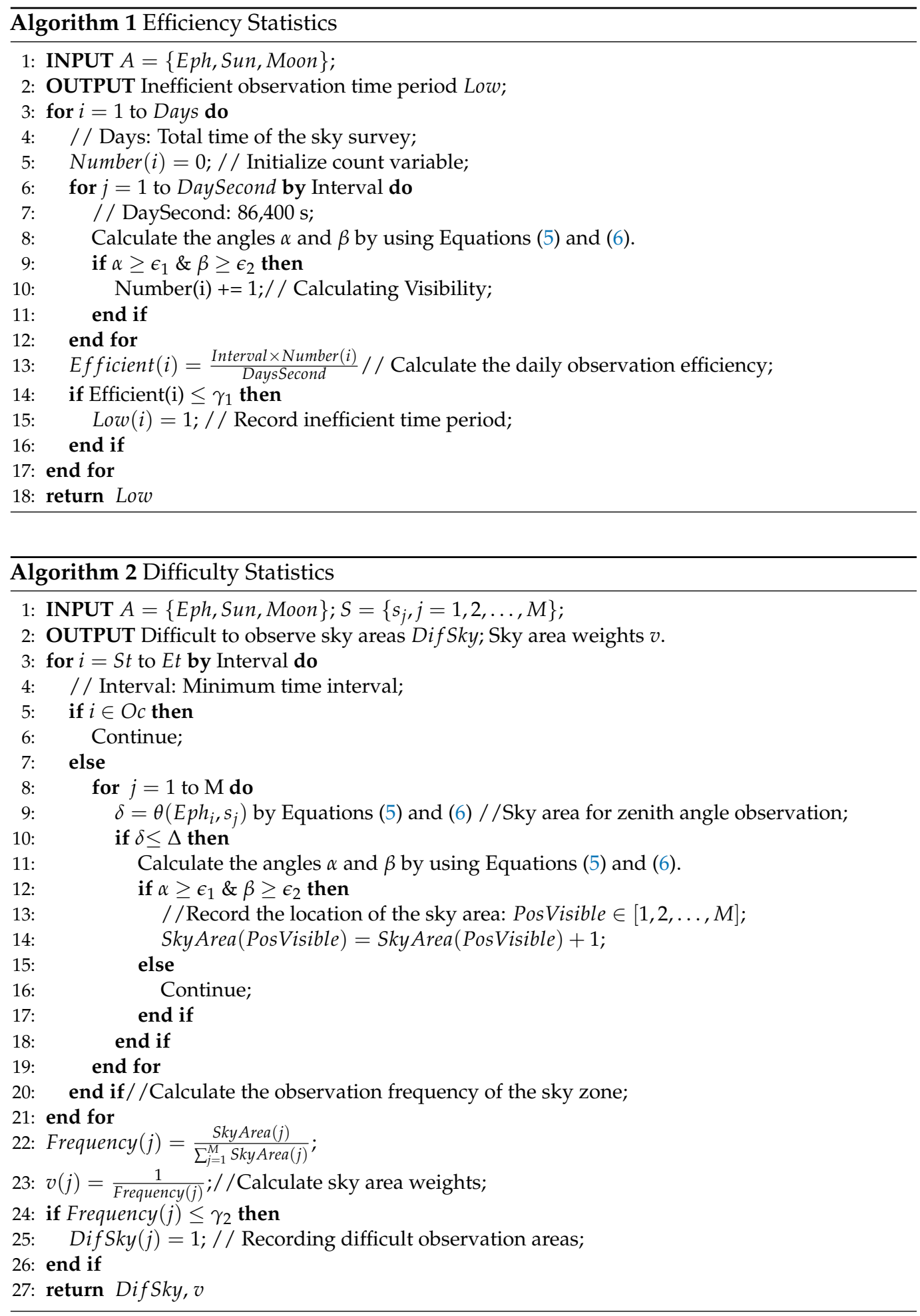

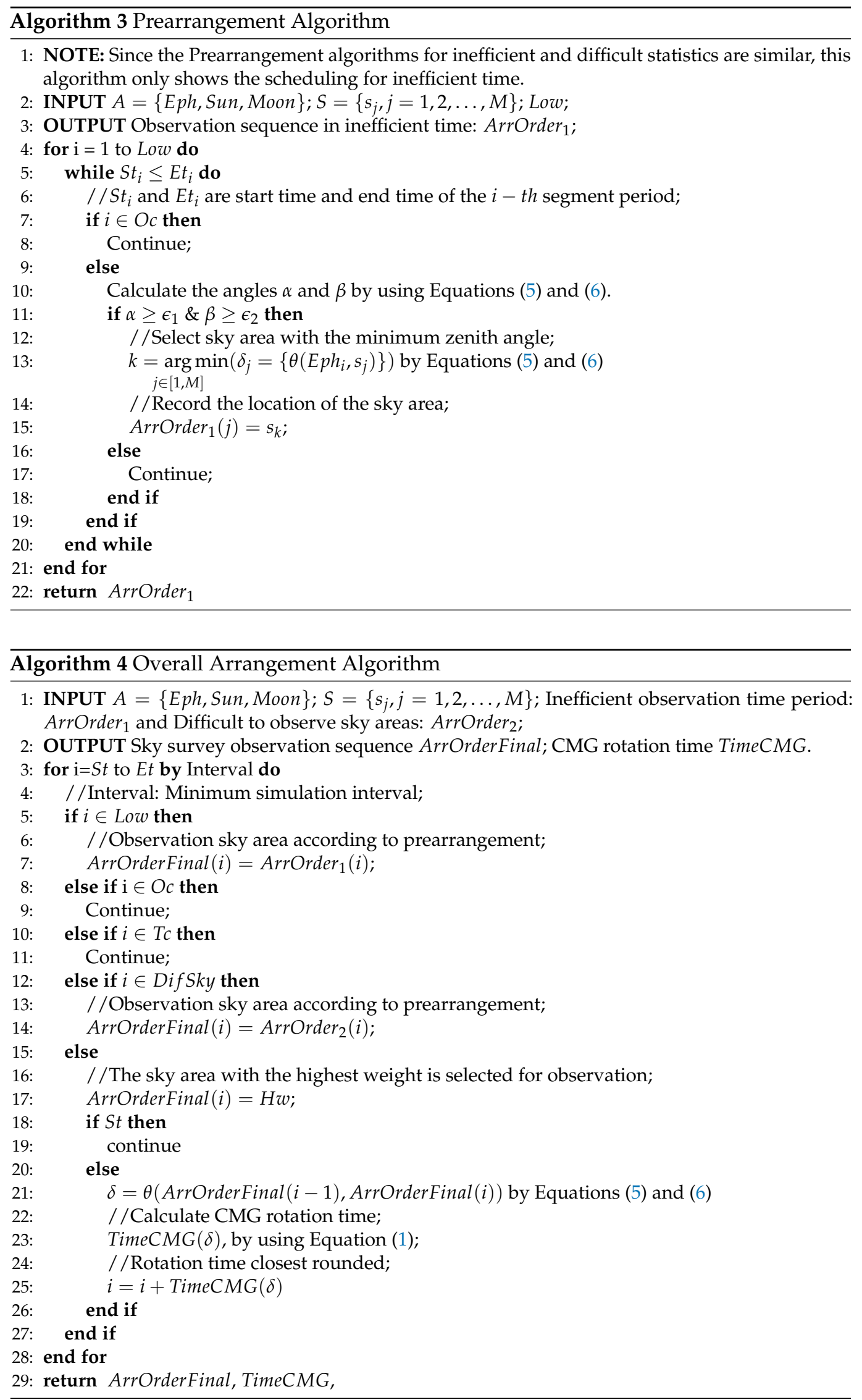


\section{Experiments and Analyses}

This section starts by providing an introduction to the basics of satellite design, including the selection of the orbit parameters, the survey area, and the selection of the algorithm parameters. Then, the sequence planned by the sky survey algorithm is statistically analyzed, including efficiency statistics, difficult sky area statistics, CMG rotation angle statistics, and coverage efficiency statistics. Finally, the observations of different parameters are compared and analyzed.

\subsection{Basic Information}

The time horizon of scheduling is from 1 January 2021 00:00 to 1 January 2030 (universal time coordinated). The experiments are performed by using an Intel (R) Core (TM) i7-7720U $2.30 \mathrm{GHz}$ CPU with 16 GB RAM under the Windows 10 operating system. The experiments and analyses will be discussed in this section.

Orbital Parameters: The spacecraft orbits in near-Earth orbit at an altitude of approximately $350-450 \mathrm{~km}$ (400 km desirable) with an orbital inclination of $42^{\circ} \sim 43^{\circ}\left(42.5^{\circ}\right.$ desirable). As part of $J_{2}$ perturbation, orbital propagation is taken into consideration, which has an effect on the RAAN by natural procession in Equation (10).

$$
\dot{\Omega}_{d}=-\frac{3}{2} J_{2} \sqrt{\mu} R_{T}^{2} a_{d}^{-\frac{7}{2}} \cos I_{d}
$$

where $\dot{\Omega}_{d}$ is the RAAN change rate of OTV on drift orbit, $J_{2}=1.086610^{-3}$ is the first zonal term of Earth, $\mu=3.98600410^{14}\left(\mathrm{~m}^{3} / \mathrm{s}^{2}\right)$ is the standard gravitational parameter, and $R_{T}=6378137(m)$ is the Earth equatorial radius. $a_{d}$ is the semi-major axis of drift orbit, and $I_{d}$ is the inclination of drift orbit, (Semimajor Axis : a, Eccentricity : e, Inclination : $i$, Argument of Perigee : $\Omega, R A A N$, Trueanomaly $: \omega,)=(6771.14 \mathrm{~km}, 0,42.5 \mathrm{deg}, 0,0,0)$. In this paper, it is assumed that the orbital model under $\mathrm{J} 2$ perturbation is adequate for the long-term sky survey planning problem [42].

Focal Plane Basical Condition: The size of each CCD is $0.22^{\circ} \times 0.2^{\circ}$. The sky survey requires full coverage of each band, and for more detailed information, refer to [28]. There is a gap between different bands in the CCD field of view, about 5 arcseconds or so. We also consider that stitching needs to require roughly $3 \%$ overlap, about 21.6 arc seconds, much larger than the gap interval. Therefore, the minimum area range should be subtracted from the overlap when performing the sky survey area division, but the gap between the filters can be disregarded. In addition, there is a strict limit to the number of angles of CMG rotation per track. It can be seen from Table 4 that if each rotation angle is less than $10^{\circ}$, $\mathrm{CMG}$ can rotate 25 times per track, and when the rotation angle is greater than $135^{\circ}$, it can only rotate 2 times.

Table 4. The maximum number of rotations corresponding to different angles of rotation in each cycle of CMG.

\begin{tabular}{ccccccccc}
\hline Angle & {$[\mathbf{5 , 1 0}]$} & {$[\mathbf{1 0 , 2 0}]$} & {$[\mathbf{2 0 , 3 5 ]}$} & {$[\mathbf{3 5 , 4 5 ]}$} & {$[\mathbf{4 5 , 7 5 ]}$} & {$[\mathbf{7 5}, \mathbf{9 0}]$} & {$[\mathbf{9 0 , 1 3 5 ]}$} & {$[\mathbf{1 3 5}, \mathbf{1 8 0}]$} \\
\hline$c_{p}$ & 25 & 15 & 10 & 8 & 5 & 4 & 3 & 2 \\
\hline
\end{tabular}

Observation Area Selection:The large scale seamless spectral survey is the main content of the sky survey. The scanning area of the survey is the J2000 flat ecliptic celestial sphere, $25 \sim 80$ degrees from north to south ecliptic, with a total area of $15,000 \mathrm{deg}^{2}$ for seamless spectral survey in Figure 3. The observation area is divided into small observation areas under the ecliptic coordinate system according to the mission objectives, and the projected coordinates of the sky area boundaries are calculated according to the telescope field of view. 


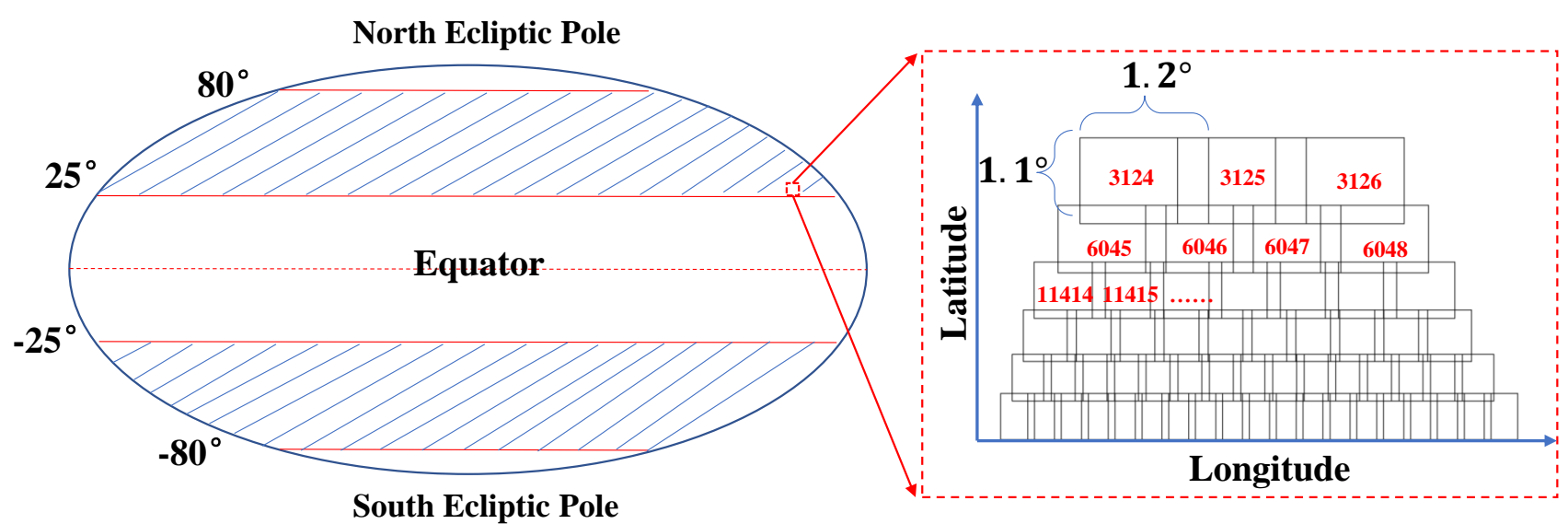

Figure 3. Diagram of sky zone division. The diagram on the left shows the sky zone division range, $\left[-80^{\circ},-25^{\circ}\right]$, and $\left[25^{\circ}, 80^{\circ}\right]$. On the right side, the dimensions of each small sky zone are shown, as well as the numbering schematic of each sky zone, which is divided into $N=17,712$ small sky zones. Ecliptic system of coordinates is used in this paper.

Algorithm Parameters: There are several important parameters in the algorithm that need to be set. First, there are two parameters $\left(\epsilon_{1}\right.$ and $\left.\epsilon_{2}\right)$ of the limitation of the tangential angle of the sunlight and the moonlight to the telescope. Then, there are the parameters for the efficiency statistics and the difficult sky area statistics. The two parameters $\left(\gamma_{1}\right.$ and $\left.\gamma_{2}\right)$ for the efficiency statistics and the difficult sky area statistics directly affect the planning results of the algorithm. Finally, there is the limiting parameter $(\Delta)$ of the zenith angle at the time of observation. The results of the parameters in the algorithm selected in the experimental part are shown in Table 5.

Table 5. Parameter values used in the experiment.

\begin{tabular}{cccccc}
\hline Parameter & $\epsilon_{1}$ & $\epsilon_{2}$ & $\gamma_{1}$ & $\gamma_{2}$ & $\Delta$ \\
\hline Value & $50^{\circ}$ & $40^{\circ}$ & 0.6 & 0.25 & $30^{\circ}$ \\
\hline
\end{tabular}

\subsection{Analysis of the Results}

Efficiency Statistics: According to the efficiency statistical method in Algorithm 1, the statistics of efficiency for the time period of the test are carried out, and the results are shown in Figure 4. From the results, it can be seen that the maximum number of days in which a complete day can be observed in 10 years is more than 160 days. The time periods with efficiency below the threshold 0.6 corresponding to these days will be prioritized using Algorithm 3.

Difficulty Statistics: The observation difficulty of the test time period sky areas is counted, and different difficulty weights are given to different sky areas. The sky areas exceeding a certain threshold are prioritized and reserved in the final complete arrangement for the advance time period. From Figure 5, it can be seen that the observation difficulty of the sky areas at high latitudes is greater, and they need to be given priority in the observation process and given greater observation weights.

CMG Rotation Angle Statistics Results: As can be seen from Figure 6, the CMG rotation angle is about 1.1 degrees in the most cases, with 4969 occurrences. The CMG rotation over 108 degrees does not exist. A normal distribution of rotation angles occurs with a mean value of about 13 degrees, indicating that most of the angles are around 13 degrees. The data of rotation angle statistics will show many rotation angles with significantly more numbers than the surrounding data shown in Figure 6a. The reason for this is that the ten-year sky survey mission is cyclical in nature, and the CMG rotation follows the greedy idea of encountering the same situation with the same angle of rotation. As can be seen in Figure 6b, the number of rotation angles with more than eight rotation angles is significantly higher than the surrounding rotation angles in the statistics of rotation angles 
below 10 degrees. Figure $6 \mathrm{c}$ shows the statistical results for intervals of 10 degrees. As can be seen, the most rotation angles are between 10 and 20 degrees, followed by the angles between 1 and 10 degrees. This indicates that the rotation angles in the ten-year mission planning are still in small rotation angles overall.

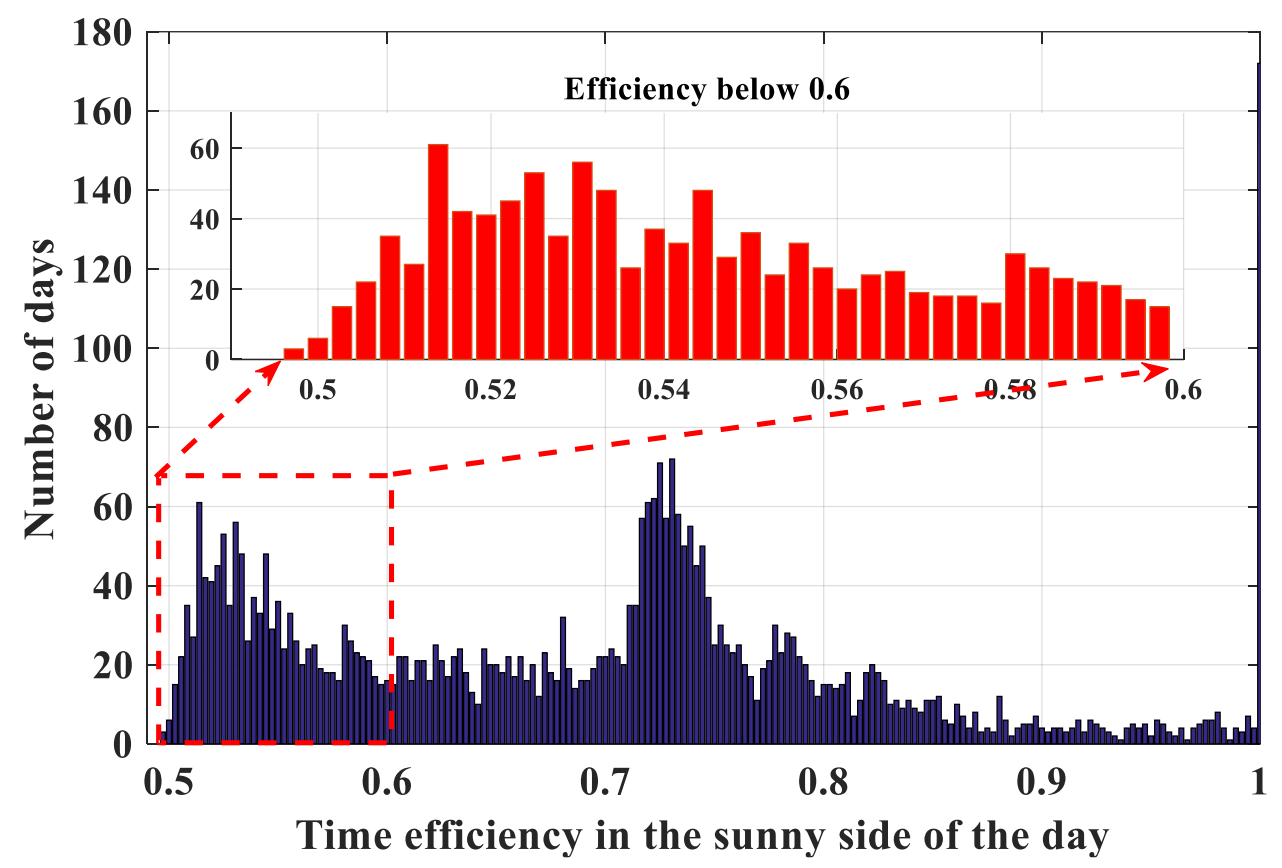

Figure 4. The results of the time efficiency statistics of the satellite sunshine zone. Horizontal axis is the ratio of the time of the day when the satellite is in the sunshine zone to the time of the whole day. Vertical axis is the statistics of the number of days. The total number of days in 10 years is 3655 days. The red bars indicate the enlargement of the fraction of time efficiency less than 0.6.

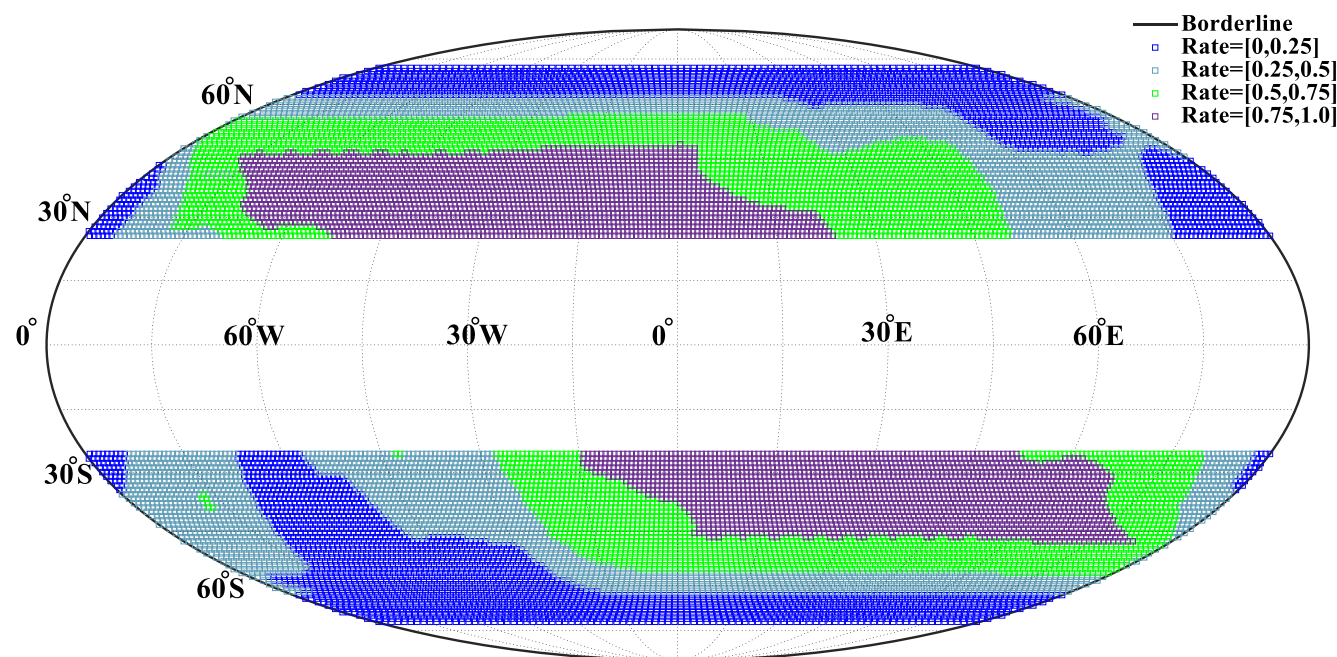

Figure 5. The result of difficult sky area statistics. Rate represents the statistical results for different quantile intervals, with smaller values representing more difficult observations and larger weighting factors. 


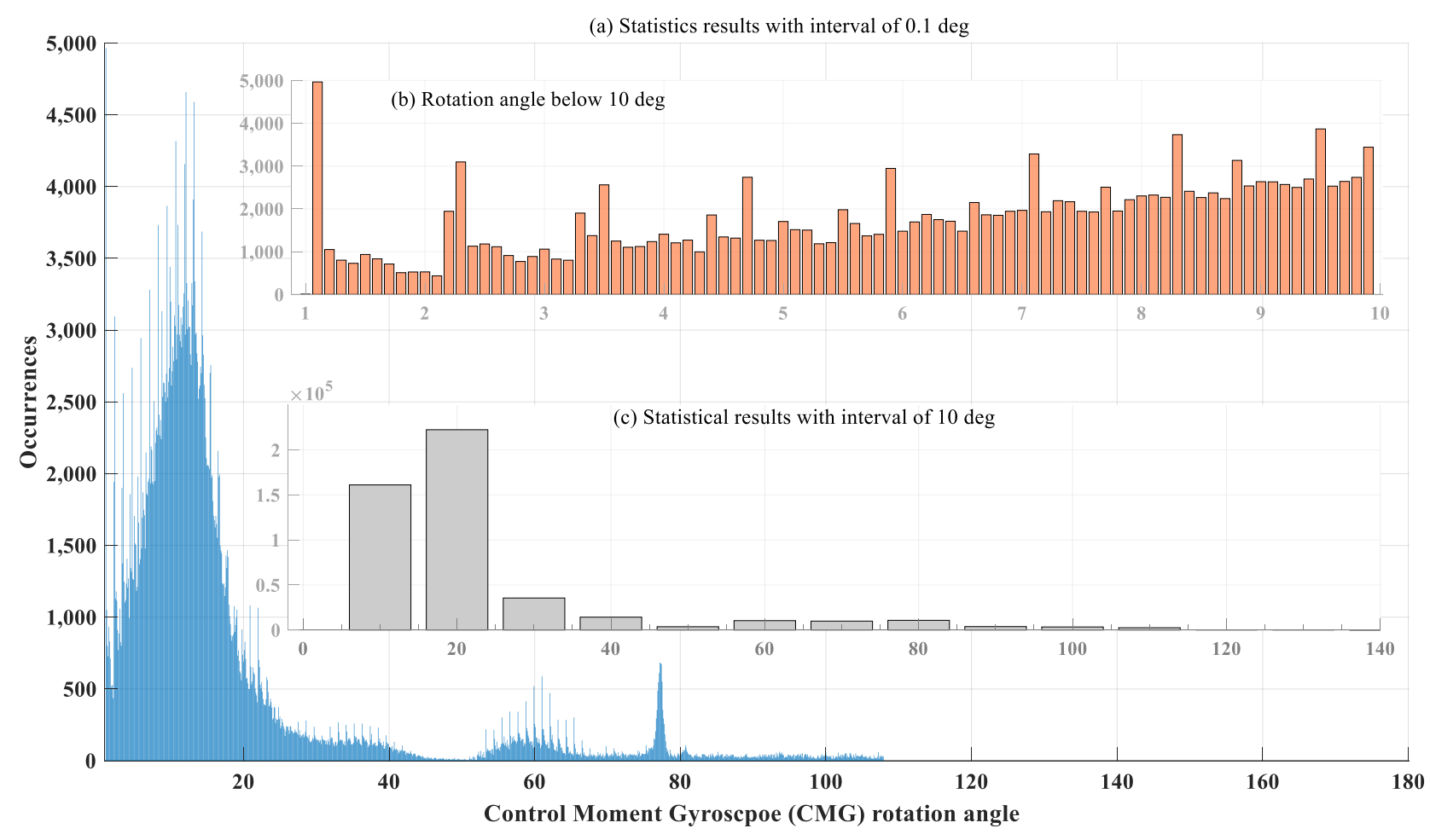

Figure 6. CMG rotation angle statistics results. (a) Represents the main graph with an angular statistic interval of $0.1^{\circ}$. (b) $\mathrm{A}$ zoomed-in display of rotation angles below $10^{\circ}$. (c) Counts the statistics at $10^{\circ}$ intervals.

It is planed to take 10 years to complete the $15,000 \mathrm{deg}^{2}$ survey, and the staged design algorithm used in this paper is able to complete the task three years ahead of schedule. The survey area in the seventh year is $15,215 \mathrm{deg}^{2}$. Figure 7 shows the growth of the survey area for different statistical intervals. From Figure 7a, it can be seen that the efficiency of the daily statistical coverage, with the red curve gradually increasing, rises more slowly in the beginning stage, which is due to the fact that the camera needs to reach 30 observations to be considered as completing a valid observation. As can be seen in Figure 8, the blue squares represent the sky areas where observations are conducted but do not reach 15 times, and this part of the sky area accounts for $82.38 \%$ of the sky area observed in the first year, while the part that completes 30 observations accounts for $14.38 \%$, further indicating that the first year mainly conducts broad observations.

During the increase of the survey area, there are many time periods when the area is not increased, and this time period represents the orbitally constrained part of the survey telescope, where the survey telescope cannot make observations, such as in the South Atlantic Anomaly region, in orbit maintenance, and when docking with the space station. The duration of this part is 120 days, which is $3.79 \%$ of the observation time. This indicates that it takes most of the time to do observations. Figure $7 \mathrm{~b}$ shows the results of the growth of the coverage area according to the monthly statistics. As can be seen from the figure, the maximum of more than $300 \mathrm{deg}^{2}$ of sky area is observed each month, and the highest observation efficiency is in the middle position, which indicates that most of the sky area in the beginning stage does not reach the number of observations, and the observation efficiency is higher in the middle stage. On the other hand, there are less unobserved sky areas in the later stage, and it takes more time to wait for the observation window to finish the observation. The area of the sky area left for observation becomes less and less, so the observation efficiency gradually decreases in the later stage.

Figure $7 \mathrm{c}$ shows the results of the growth of the covered area according to the annual statistics. As can be seen, a maximum of more than $2600 \mathrm{deg}^{2}$ of the sky area is observed in the third and fourth years. The eighth year has a total observation area of more than $500 \mathrm{deg}^{2}$ in nine months, which is $75 \%$ of the time. However, it is $25 \%$ of the time of the 
first year, indicating that the eighth year is in the stage of replenishing the sky area. In addition, the areas that are difficult to observe in the previous years are replenished with observations in the ninth year. From Figure $9 g$, it can be seen that the area observed in the ninth year is very small and mainly concentrated in the sky areas that are difficult to observe in the previous years.

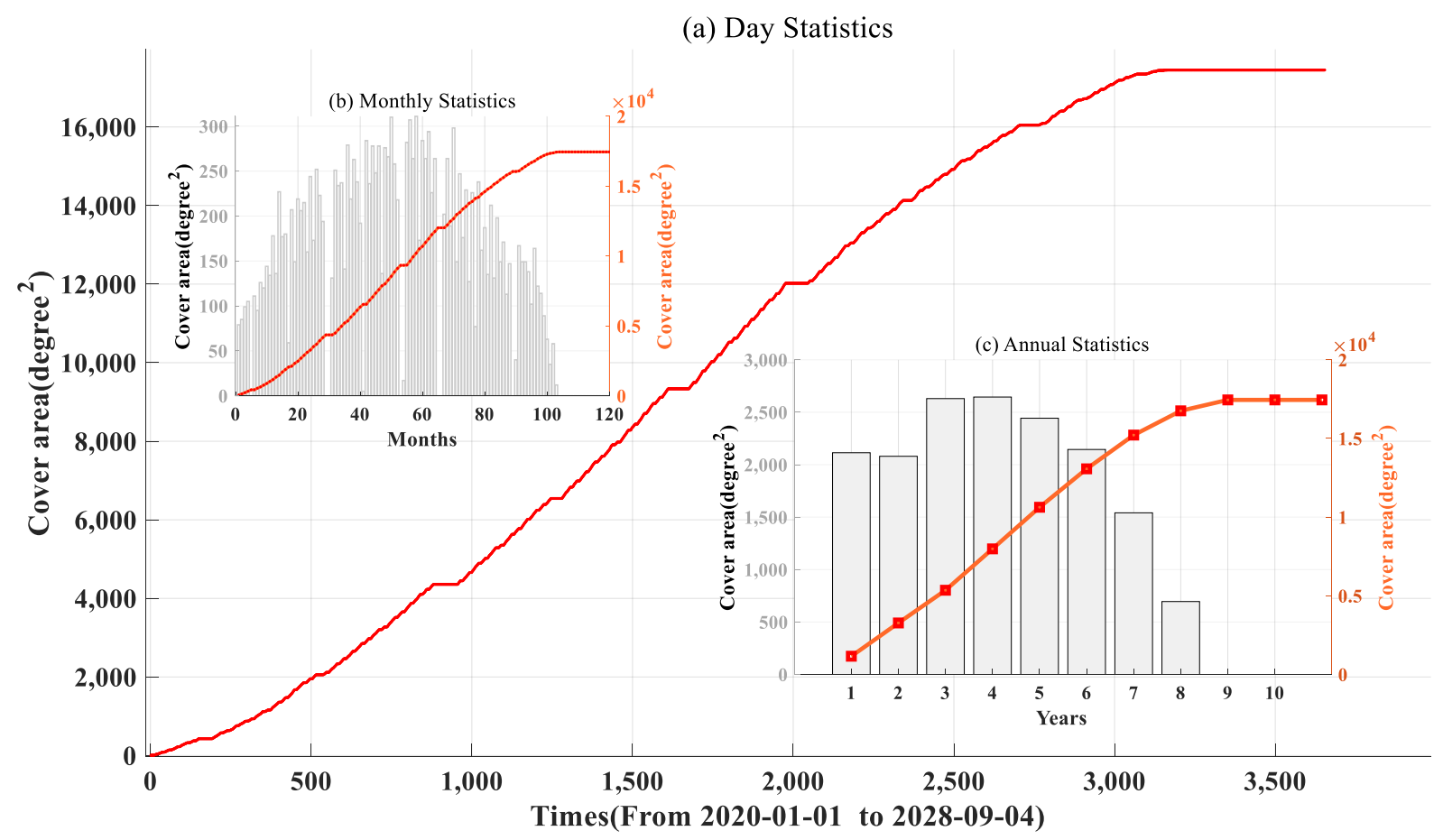

Figure 7. Statistical results of sky survey coverage efficiency. (a) The cumulative statistical results according to the daily coverage efficiency. (b) The cumulative coverage efficiency according to the monthly statistics and the results of each month. (c) The cumulative results of the coverage efficiency according to the annual statistics and the data of each year.

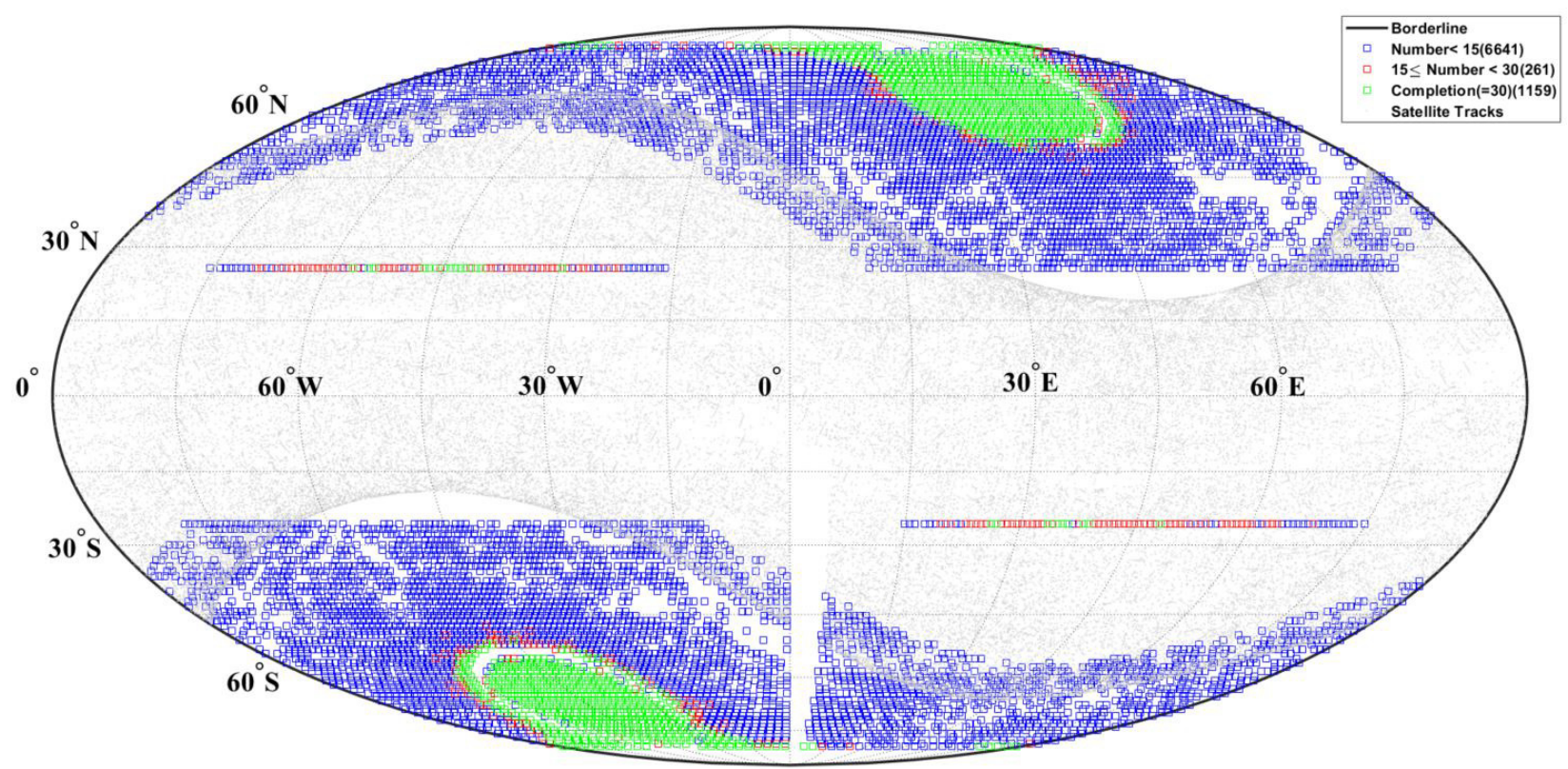

Figure 8. The results of the first year of sky surveys, with different colors representing different numbers of observations. 


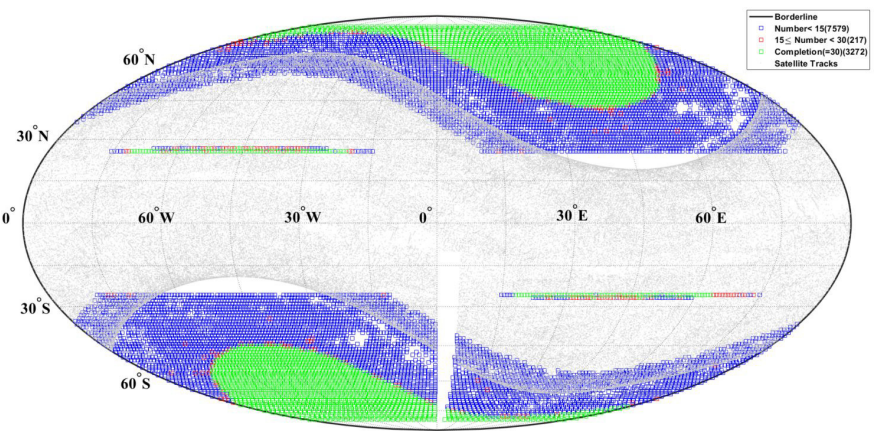

(a) Second year (Cover area:3,272 $\mathrm{deg}^{2}$ )

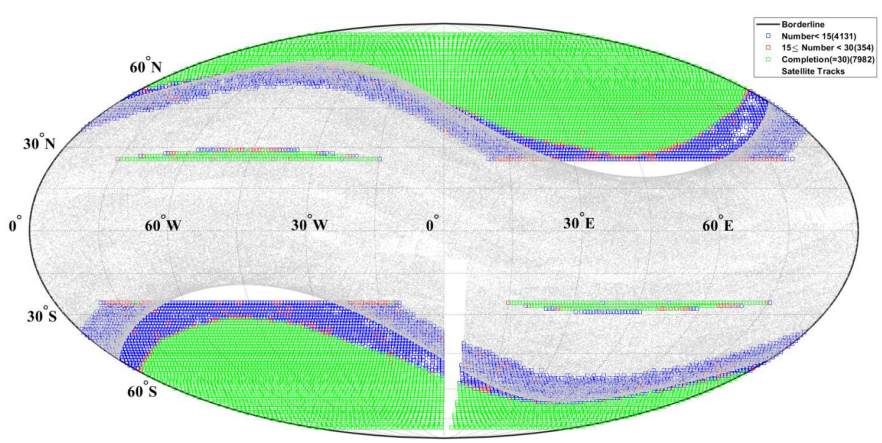

(c) Fourth year (Cover area:7,982 deg²)

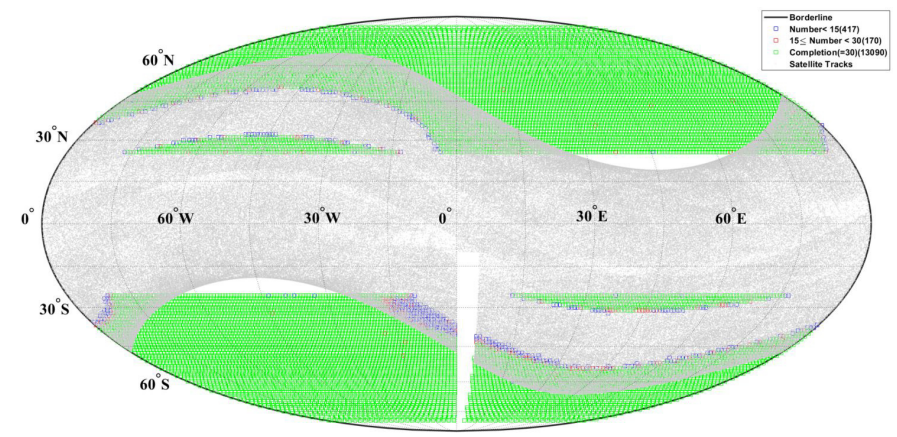

(e) Sixth year (Cover area:13,090 deg²)

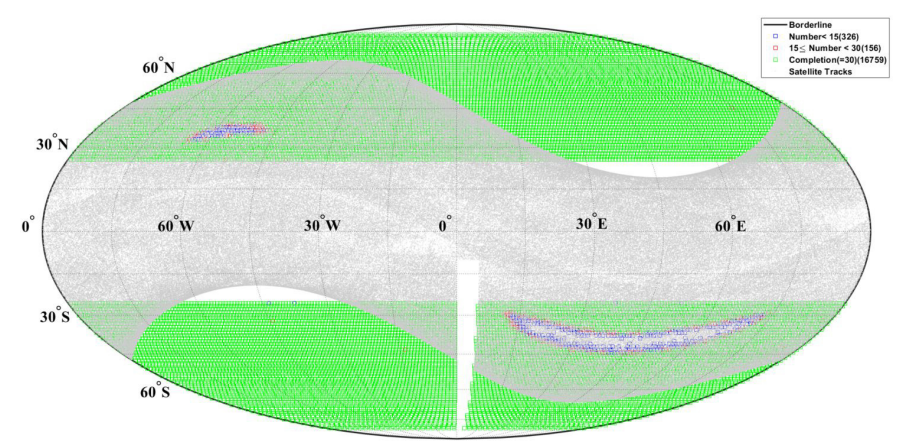

(g) Eighth year (Cover area:16,759 deg²)

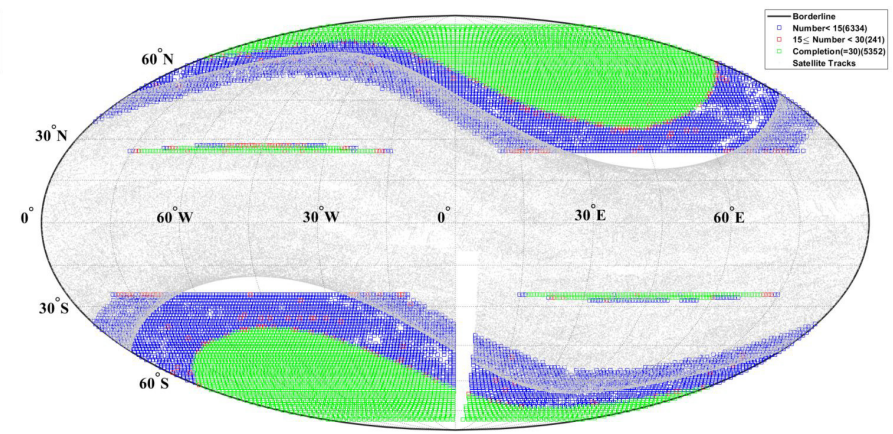

(b) Third year (Cover area:5,352 $\mathrm{deg}^{2}$ )

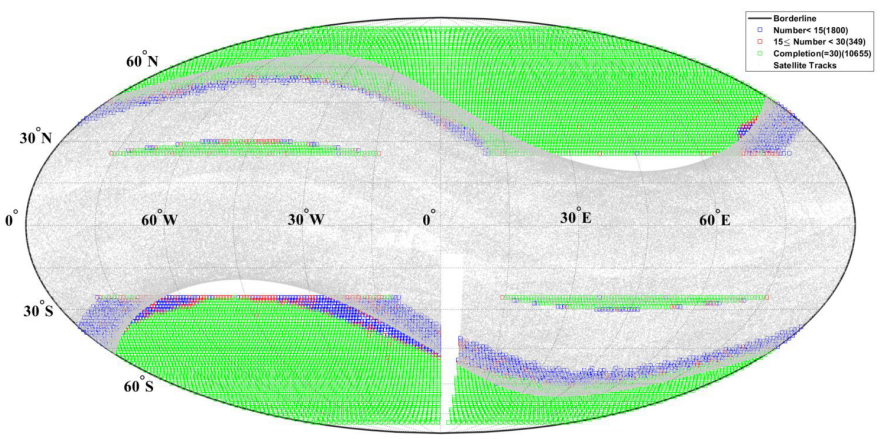

(d) Fifth year (Cover area:10,655 deg ${ }^{2}$ )

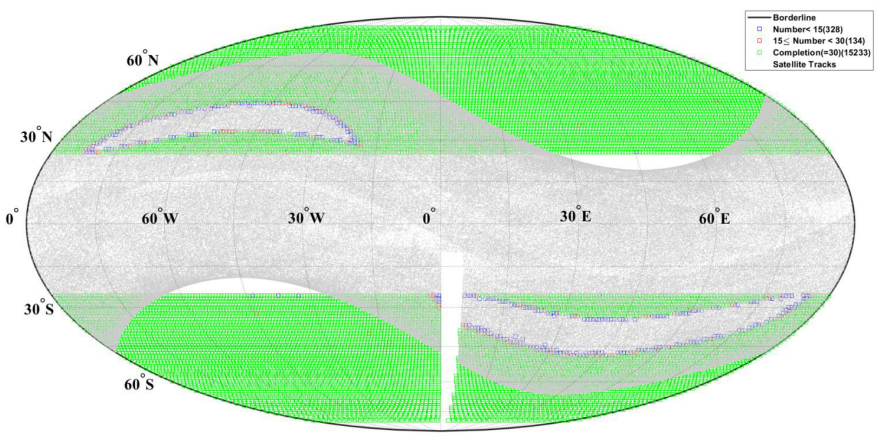

(f) Seventh year (Cover area:15,233 deg²)

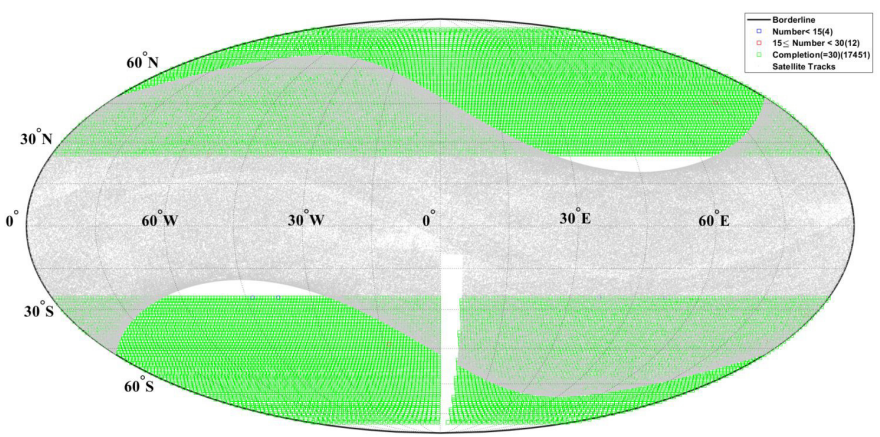

(h) Ninth year (Cover area:17,451 deg')

Figure 9. Presentation of observation results from the second year to the ninth year with parameter $\gamma_{1}=0.6$.

Figures 8 and 9 show the display of the effect of the sky survey observations from the second to ninth year. The two elliptical sky regions with dimensions near the South Pole and the North Pole are observed first, because they are found to be more difficult to observe after several planning sessions. The weighting factors of the regions are increased, so that they are observed first. 


\subsection{Experimentation of Algorithm Parameters}

Three of the five parameters shown in Table 5 are designed according to the satellite orbit, and two parameters are related to the algorithm design. The two parameters of the algorithm design are set differently, and the results of the sky survey planning are different. In order to study the specific effect of parameter settings on the sky survey effect, this section conducts experiments on the controlling efficiency parameter $\gamma_{1}$.

The results of the sky survey area with different parameters are shown in Figure 10. It can be seen that the first to fifth years of the sky survey are slow with the parameter $\left(\gamma_{1}=0.7\right)$, increasing the area by about $1600 \mathrm{deg}^{2}$ at most. The sixth year increases the most, about $5200 \mathrm{deg}^{2}$ of the sky survey area, indicating that some of the sky areas accumulated in the previous years with an insufficient number of observations are observed a lot in the sixth year. This breaks through the single-year observation area of more than $5000 \mathrm{deg}^{2}$. The case of parameter 0.7 is slightly different from the case of parameter 0.6. Since the increase of the efficiency parameter makes it so that the sky areas that are not prioritized in the previous years are scheduled, it can be seen from Figure 10a that the observed sky area is more homogeneous compared with the case of parameter 0.6. In addition, the increase in observations is slow before the sixth year, and a rapid increase occurs in the sixth year. The eighth year of the resultant observations can complete $16,492 \mathrm{deg}^{2}$ of the sky area. For the case of parameter 0.6 , it is a slow-growing type overall, with a maximum increase of no more than $3000 \mathrm{deg}^{2}$ in a single year, illustrating the stable growth.

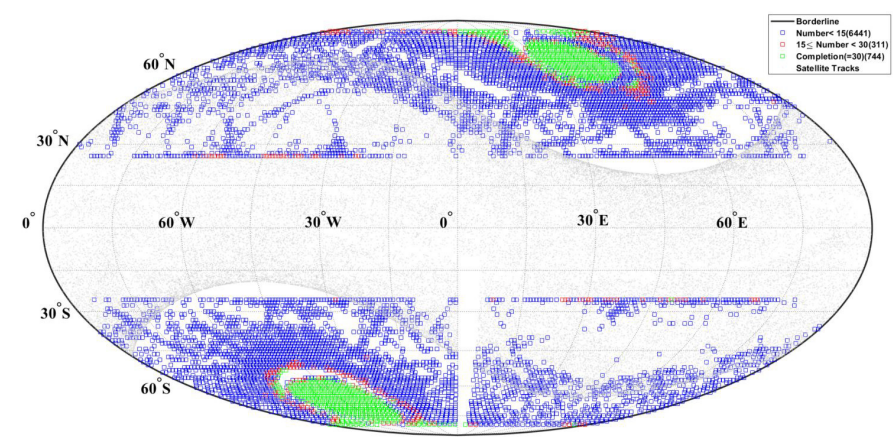

(a) First year (Cover area:744 deg ${ }^{2}$ )

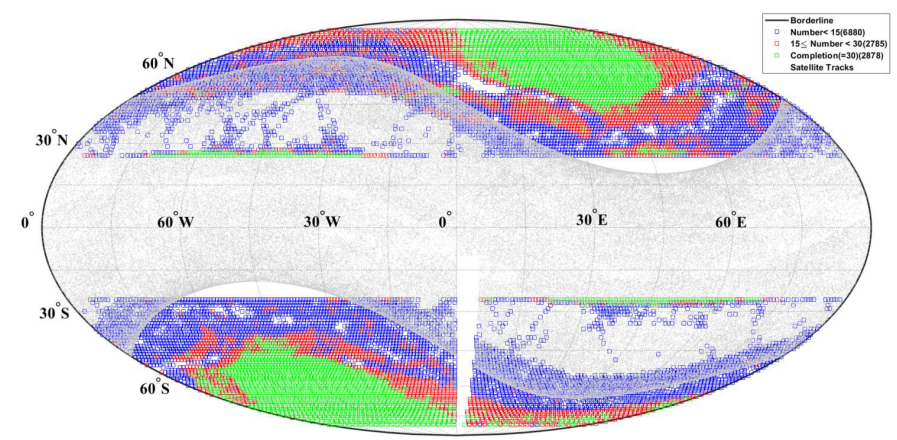

(c) Third year (Cover area:2,878 $\mathrm{deg}^{2}$ )

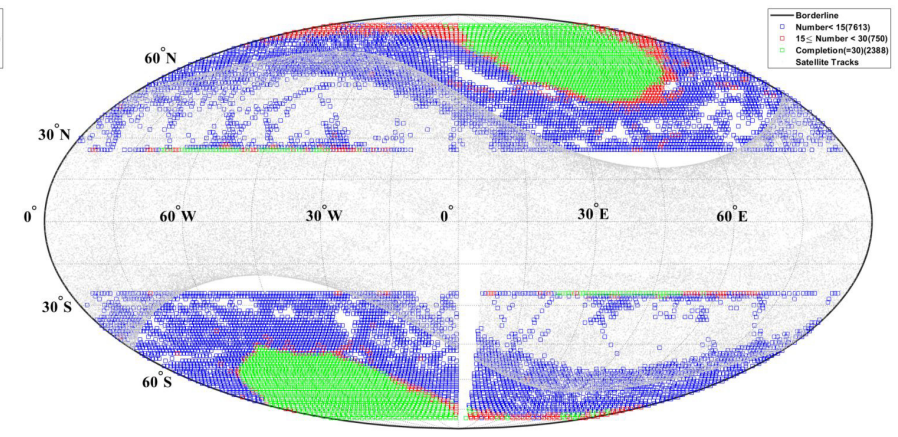

(b) Second year (Cover area:2,388 deg²)

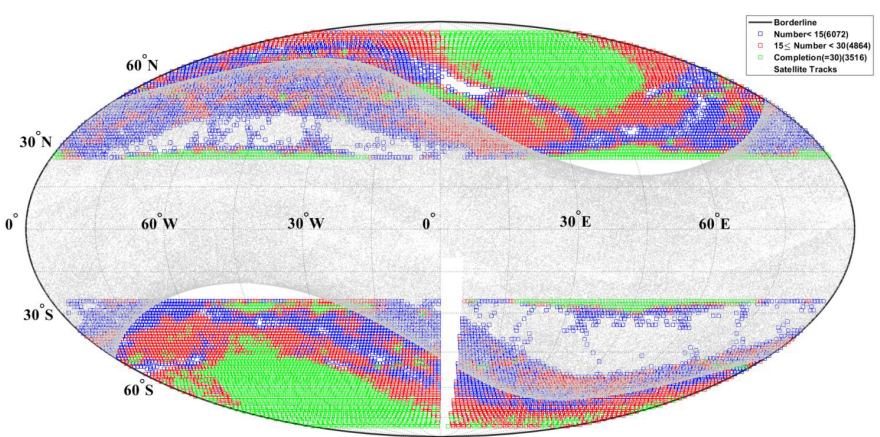

(d) Fourth year (Cover area:3,516 deg${ }^{2}$ )

Figure 10. Cont. 


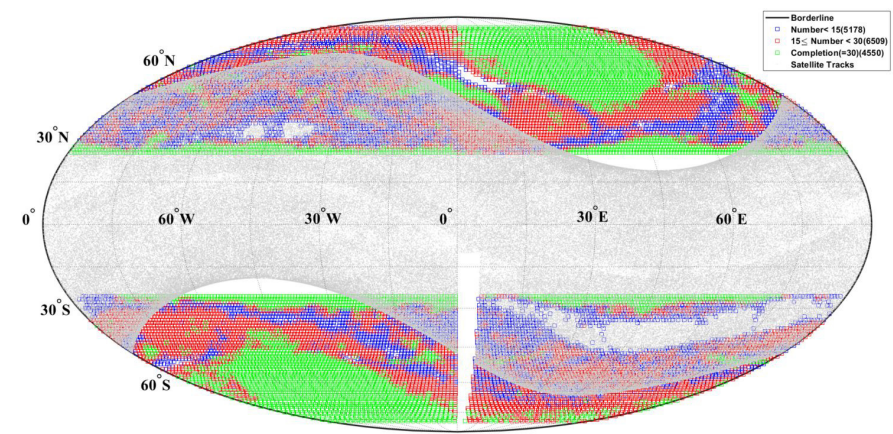

(e) Fifth year (Cover area:4,550 deg²)

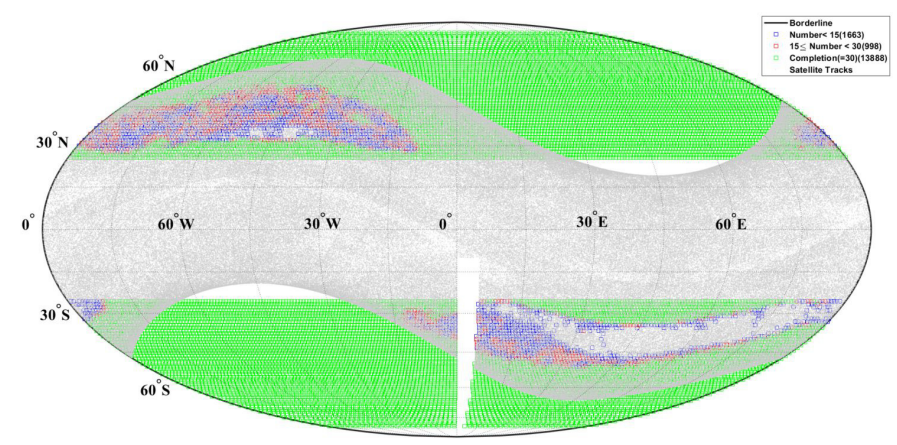

(g) Seventh year (Cover area:13,888 deg²)

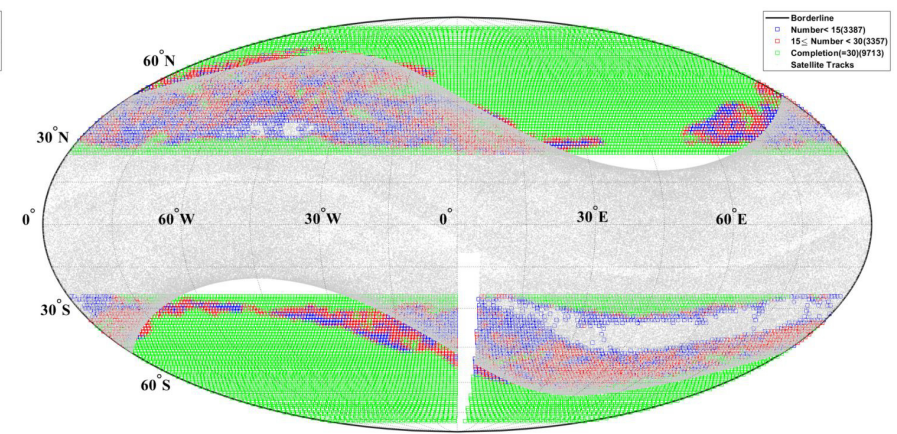

(f) Sixth year (Cover area:9,713 $\mathrm{deg}^{2}$ )

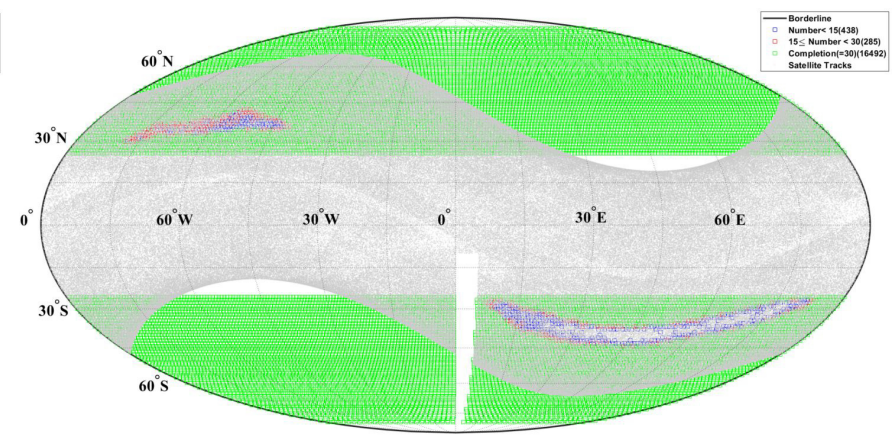

(h) Eighth year (Cover area:16,492 deg²)

Figure 10. Presentation of observation results from the first year to the eighth year with parameter $\gamma_{1}=0.7$.

\section{Discussion}

In this paper, we systematically analyze various conditions of the sky survey observation planning task and then establish a multi-objective optimization model with several constraints. An efficient planning strategy is an important part of the sky survey planning mission. If satellites in fixed orbits are left free to observe without observation sequence optimization, this will lead to an extremely low observation efficiency and even fail to meet the observation requirements during the lifetime of the satellite. Thus, the optimization of the sky survey observation sequence is especially important. For small-scale and shorttime surveys, there are more mature heuristic algorithms that can solve the problem well. However, for wide and deep sky surveys, the heuristic algorithm seems incompetent and powerless. The core idea of the proposed algorithm is to obtain the prearranged sequence from two perspectives: the inefficient time period and the difficult observation sky area.

In order to verify the correctness of the model and the reliability of the algorithm in this paper, a wide and deep sky survey mission with more than a 15,000 deg area over a 10 -year period is selected for the experiment, which has an essential improvement over the results of existing algorithms using homemade or small-scale datasets. According to the experimental results, it can be seen that the 10-year observation period Staged Design Algorithm proposed in this paper can complete the effective observation of sky areas and achieve the target of $15,000 \mathrm{deg}^{2}$ of sky survey three years ahead of schedule. The important parameters involved in the algorithm, such as the analysis of the inefficiency threshold, are also analyzed.

\section{Conclusions}

To solve the problems of incompleteness and deviation from the actual engineering tasks of existing studies on telescope survey optimization, this paper establishes a multiobjective model with complex constraints based on existing survey planning studies, which are split into observation constraints and orbit constraints, and designs a multi-stage greedy algorithm to solve it. The model is solved by combining the actual design orbit parameters 
of the China Space Station Telescope and the survey objectives. The results show that the $15,000 \mathrm{deg}^{2}$ survey target can be completed three years earlier using the Staged Design Algorithm proposed in this paper, indicating that the algorithm can actually improve the survey efficiency.

The Staged Design Algorithm proposed in this paper can accomplish the task of wide and deep sky surveys very well, but there are still some improvements for enhancement. In this paper, only a single class of sky survey observations is performed for a single time, and depth observations and extremely deep observations can be considered for future work. In addition, the greedy algorithm is used in the process of prearranging inefficient time intervals and difficult observation sky areas. Although the algorithm reduces the complexity, it does not achieve the optimal observation of short time intervals, and should be further improved in the future by combining it with the algorithm related to Earth observation.

Author Contributions: Conceptualization, K.H., H.W., W.Z., and Y.X.; Methodology, K.H., S.H. and W.Z.; Software, K.H. and W.Z.; Supervision, H.W., Y.X. and S.H.; Validation, K.H. and S.H.; Visualization, K.H., H.W. and W.Z.; Writing-original draft, K.H.; Writing-review and editing, K.H. and Y.X. All authors have read and agreed to the published version of the manuscript.

Funding: This research received no external funding.

Institutional Review Board Statement: Not applicable.

Informed Consent Statement: Informed consent was obtained from all subjects involved in the study.

Data Availability Statement: Not applicable.

Conflicts of Interest: The authors declare no conflict of interest.

\section{References}

1. Bock, D.J.; Large, M.; Sadler, E.M. SUMSS: A wide-field radio imaging survey of the southern sky. I. Science goals, survey design, and instrumentation. Astron. J. 1999, 117, 1578. [CrossRef]

2. Lawrence, A.; Warren, S.; Almaini, O.; Edge, A.; Hambly, N.; Jameson, R.; Lucas, P.; Casali, M.; Adamson, A.; Dye, S.; et al. The UKIRT infrared deep sky survey (UKIDSS). Mon. Not. R. Astron. Soc. 2007, 379, 1599-1617. [CrossRef]

3. Riess, A.G.; Filippenko, A.V.; Challis, P.; Clocchiatti, A.; Diercks, A.; Garnavich, P.M.; Gilliland, R.L.; Hogan, C.J.; Jha, S.; Kirshner, R.P.; et al. Observational evidence from supernovae for an accelerating universe and a cosmological constant. Astron. J. 1998, 116, 1009. [CrossRef]

4. Astier, P.; Guy, J.; Regnault, N.; Pain, R.; Aubourg, E.; Balam, D.; Basa, S.; Carlberg, R.; Fabbro, S.; Fouchez, D.; et al. The Supernova Legacy Survey: Measurement of, and w from the first year data set. Astron. Astrophys. 2006, 447, 31-48. [CrossRef]

5. Fruchter, A.; Levan, A.; Strolger, L.; Vreeswijk, P.; Thorsett, S.; Bersier, D.; Burud, I.; Cerón, J.C.; Castro-Tirado, A.; Conselice, C.; et al. Long $\gamma$-ray bursts and core-collapse supernovae have different environments. Nature 2006, 441, $463-468$. [CrossRef]

6. Perlmutter, S.; Gabi, S.; Goldhaber, G.; Goobar, A.; Groom, D.; Hook, I.; Kim, A.; Kim, M.; Lee, J.; Pain, R.; et al. Measurements* of the Cosmological Parameters $\Omega$ and $\Lambda$ from the First Seven Supernovae at $\mathrm{z} \geq 0.35$. Astrophys. J. 1997, 483, 565. [CrossRef]

7. Fukugita, M.; Shimasaku, K.; Ichikawa, T.; Gunn, J. The Sloan Digital Sky Survey Photometric System. Astron. J. 1996, 111, 1748. [CrossRef]

8. York, D.G.; Adelman, J.; Anderson, J.E., Jr.; Anderson, S.F.; Annis, J.; Bahcall, N.A.; Bakken, J.; Barkhouser, R.; Bastian, S.; Berman, E.; et al. The sloan digital sky survey: Technical summary. Astron. J. 2000, 120, 1579. [CrossRef]

9. Abazajian, K.N.; Adelman-McCarthy, J.K.; Agüeros, M.A.; Allam, S.S.; Prieto, C.A.; An, D.; Anderson, K.S.; Anderson, S.F.; Annis, J.; Bahcall, N.A.; et al. The seventh data release of the Sloan Digital Sky Survey. Astrophys. J. Suppl. Ser. 2009, 182, 543. [CrossRef]

10. Abell, P.A.; Allison, J.; Anderson, S.F.; Andrew, J.R.; Angel, J.R.P.; Armus, L.; Arnett, D.; Asztalos, S.; Axelrod, T.S.; Bailey, S.; et al. Lsst science book, version 2.0. arXiv 2009, arXiv:0912.0201.

11. Marshall, P.; Anguita, T.; Bianco, F.B.; Bellm, E.C.; Brandt, N.; Clarkson, W.; Connolly, A.; Gawiser, E.; Ivezic, Z.; Jones, L.; et al. Science-driven optimization of the LSST observing strategy. arXiv 2017, arXiv:1708.04058.

12. Ivezić, Ž.; Kahn, S.M.; Tyson, J.A.; Abel, B.; Acosta, E.; Allsman, R.; Alonso, D.; AlSayyad, Y.; Anderson, S.F.; Andrew, J.; et al. LSST: From science drivers to reference design and anticipated data products. Astrophys. J. 2019, 873, 111. [CrossRef]

13. Holtzman, J.A.; Hester, J.J.; Casertano, S.; Trauger, J.T.; Watson, A.M.; Ballester, G.E.; Burrows, C.J.; Clarke, J.T.; Crisp, D.; Evans, R.W.; et al. The performance and calibration of WFPC2 on the Hubble Space Telescope. Publ. Astron. Soc. Pac. 1995, 107, 156. [CrossRef] 
14. Freedman, W.L.; Madore, B.F.; Gibson, B.K.; Ferrarese, L.; Kelson, D.D.; Sakai, S.; Mould, J.R.; Kennicutt, R.C., Jr.; Ford, H.C.; Graham, J.A.; et al. Final results from the Hubble Space Telescope key project to measure the Hubble constant. Astrophys. J. 2001, 553, 47. [CrossRef]

15. Scoville, N.; Abraham, R.; Aussel, H.; Barnes, J.; Benson, A.; Blain, A.; Calzetti, D.; Comastri, A.; Capak, P.; Carilli, C.; et al. COSMOS: Hubble space telescope observations. Astrophys. J. Suppl. Ser. 2007, 172, 38. [CrossRef]

16. Laureijs, R.; Amiaux, J.; Arduini, S.; Augueres, J.L.; Brinchmann, J.; Cole, R.; Cropper, M.; Dabin, C.; Duvet, L.; Ealet, A.; et al. Euclid definition study report. arXiv 2011, arXiv:1110.3193.

17. Amiaux, J.; Scaramella, R.; Mellier, Y.; Altieri, B.; Burigana, C.; Da Silva, A.; Gomez, P.; Hoar, J.; Laureijs, R.; Maiorano, E.; et al. Euclid Mission: Building of a reference survey. In Space Telescopes and Instrumentation 2012: Optical, Infrared, and Millimeter Wave; International Society for Optics and Photonics: Washington, DC, USA, 2012; Volume 8442, p. 84420Z.

18. Amendola, L.; Appleby, S.; Avgoustidis, A.; Bacon, D.; Baker, T.; Baldi, M.; Bartolo, N.; Blanchard, A.; Bonvin, C.; Borgani, S.; et al. Cosmology and fundamental physics with the Euclid satellite. Living Rev. Relativ. 2018, 21, 2. [CrossRef]

19. Spergel, D.N.; Verde, L.; Peiris, H.V.; Komatsu, E.; Nolta, M.; Bennett, C.L.; Halpern, M.; Hinshaw, G.; Jarosik, N.; Kogut, A.; et al. First-year Wilkinson Microwave Anisotropy Probe (WMAP)* observations: Determination of cosmological parameters. Astrophys. J. Suppl. Ser. 2003, 148, 175. [CrossRef]

20. Komatsu, E.; Dunkley, J.; Nolta, M.; Bennett, C.; Gold, B.; Hinshaw, G.; Jarosik, N.; Larson, D.; Limon, M.; Page, L.; et al. Five-year wilkinson microwave anisotropy probe* observations: Cosmological interpretation. Astrophys. J. Suppl. Ser. 2009, 180, 330. [CrossRef]

21. Bennett, C.L.; Larson, D.; Weiland, J.L.; Jarosik, N.; Hinshaw, G.; Odegard, N.; Smith, K.; Hill, R.; Gold, B.; Halpern, M.; et al. Nine-year Wilkinson Microwave Anisotropy Probe (WMAP) observations: final maps and results. Astrophys. J. Suppl. Ser. 2013, 208, 20. [CrossRef]

22. Bevis, N.; Hindmarsh, M.; Kunz, M.; Urrestilla, J. CMB power spectra from cosmic strings: Predictions for the Planck satellite and beyond. Phys. Rev. D 2010, 82, 065004. [CrossRef]

23. Salvatelli, V.; Marchini, A.; Lopez-Honorez, L.; Mena, O. New constraints on Coupled Dark Energy from the Planck satellite experiment. Phys. Rev. D 2013, 88, 023531. [CrossRef]

24. Green, J.; Schechter, P.; Baltay, C.; Bean, R.; Bennett, D.; Brown, R.; Conselice, C.; Donahue, M.; Fan, X.; Gaudi, B.; et al. Wide-field infrared survey telescope (WFIRST) final report. arXiv 2012, arXiv:1208.4012.

25. Spergel, D.; Gehrels, N.; Breckinridge, J.; Donahue, M.; Dressler, A.; Gaudi, B.; Greene, T.; Guyon, O.; Hirata, C.; Kalirai, J.; et al. Wide-field infrared survey telescope-astrophysics focused telescope assets WFIRST-AFTA final report. arXiv 2013, arXiv:1305.5422.

26. Fitzmaurice, J.; Bédard, D.; Lee, C.H.; Seitzer, P. Detection and correlation of geosynchronous objects in NASA's Wide-field Infrared Survey Explorer images. Acta Astronaut. 2021, 183, 176-198. [CrossRef]

27. Zhan, H. Consideration for a large-scale multi-color imaging and slitless spectroscopy survey on the Chinese space station and its application in dark energy research. Sci. Sin. Phys. Mech. Astron. 2011, 41, 1441-1447. [CrossRef]

28. Zhan, H. The wide-field multiband imaging and slitless spectroscopy survey to be carried out by the Survey Space Telescope of China Manned Space Program. Chin. Sci. Bull. 2021, 66, 1290-1298. [CrossRef]

29. Johnston, M.D.; Giuliano, M.E. Multi-Objective Scheduling for Space Science Missions. J. Adv. Comput. Intell. Intell. Inform. 2011, 15, 1140-1148. [CrossRef]

30. Liu, W.; Lin, B.J. Intelligent Model of Astronomical Satellite Using GA for Scanning the Celestial Sphere. J. Syst. Simul. 2007, 3, 654-656.

31. Jin, Z.; Liu, C. Modeling and Simulation of Large Sky Area and Observation for onboard Sky Survey Multicolor Imaging Telescope. Comput. Technol. Dev. 2013, 23, 155-160. (In chinese)

32. Wang, X.; Lu, H. 0-1 Linear Programming for CNEOST(China Near Earth Object Survey Telescope) Survey Scheduling. Acta Astron. Sin. 2015, 56, 391-398.

33. Huang, Y.; Qu, J.; Jia, S.; Ma, X.; Song, L.; Chen, L.; Zhang, W. Long-term planning algorithm for the HXMT mission. Chin. J. Space Sci. 2017, 37, 766-772.

34. Cenko, S.B.; Fox, D.B.; Moon, D.S.; Harrison, F.A.; Kulkarni, S.; Henning, J.R.; Guzman, C.D.; Bonati, M.; Smith, R.M.; Thicksten, R.P.; et al. The automated Palomar 60 inch telescope. Publ. Astron. Soc. Pac. 2006, 118, 1396. [CrossRef]

35. Kubanek, P. Genetic algorithm for robotic telescope scheduling. arXiv 2010, arXiv:1002.0108.

36. Johnston, M.D.; Miller, G. Spike: Intelligent scheduling of hubble space telescope observations. Intell. Sched. 1994, $391-422$.

37. Wu, G.; Liu, J.; Ma, M.; Qiu, D. A two-phase scheduling method with the consideration of task clustering for earth observing satellites. Comput. Oper. Res. 2013, 40, 1884-1894. [CrossRef]

38. Wang, J.; Zhu, X.; Qiu, D.; Yang, L.T. Dynamic scheduling for emergency tasks on distributed imaging satellites with task merging. IEEE Trans. Parallel Distrib. Syst. 2013, 25, 2275-2285. [CrossRef]

39. Gledhill, J. Aeronomic effects of the South Atlantic anomaly. Rev. Geophys. 1976, 14, 173-187. [CrossRef]

40. Maling, D.H. Coordinate Systems and Map Projections; Elsevier: Amsterdam, The Netherlands, 2013.

41. Snyder, J.P. MAP Projections: A Working Manual; United States Government Printing Office: Washington, DC, USA, 1987; pp. 182-190.

42. Vallado, D.A. Fundamentals of Astrodynamics and Applications. Springer Science \& Business Media: New York, NY, USA, 2001; Volume 12. 